\title{
Groundwater Quality and Simulation of Sources of Water to Wells in the Marsh Creek Valley at the U.S. Geological Survey Northern Appalachian Research Laboratory, Tioga County, Pennsylvania
}

Scientific Investigations Report 2012-5042 
Cover. Photograph of Marsh Creek valley looking west from the Northern Appalachian Research Laboratory, November 2, 2010. (Photograph courtesy of Linda Jo Kraemer) 


\section{Groundwater Quality and Simulation of Sources of Water to Wells in the Marsh Creek Valley at the U.S. Geological Survey Northern Appalachian Research Laboratory, Tioga County, Pennsylvania}

By Dennis W. Risser and Kevin J. Breen

Scientific Investigations Report 2012-5042 


\title{
U.S. Department of the Interior \\ KEN SALAZAR, Secretary \\ U.S. Geological Survey \\ Marcia K. McNutt, Director
}

\author{
U.S. Geological Survey, Reston, Virginia: 2012
}

For more information on the USGS - the Federal source for science about the Earth, its natural and living resources, natural hazards, and the environment, visit http://www.usgs.gov or call 1-888-ASK-USGS.

For an overview of USGS information products, including maps, imagery, and publications, visit http://www.usgs.gov/pubprod

To order this and other USGS information products, visit http://store.usgs.gov

Any use of trade, product, or firm names is for descriptive purposes only and does not imply endorsement by the U.S. Government.

Although this report is in the public domain, permission must be secured from the individual copyright owners to reproduce any copyrighted materials contained within this report.

Suggested citation:

Risser, D.W., and Breen, K.J., 2012, Groundwater quality and simulation of sources of water to wells in the Marsh Creek valley at the U.S. Geological Survey Northern Appalachian Research Laboratory, Tioga County, Pennsylvania: U.S. Geological Survey Scientific Investigations Report 2012-5042, 41 p., at http://pubs.usgs.gov/sir/2012/5042/. 


\section{Contents}

Abstract Introduction
Purpose and Scope
Previous Investigations.
Study Methods
Groundwater-Quality Sampling
Collection and Analysis of Samples Assurance
$\quad$ Spdates to the Model.

\section{Figures}

1. Map showing the location of the study area in the Marsh Creek valley, near Wellsboro, Tioga County, Pennsylvania.

2. Photograph showing Northern Appalachian Research Laboratory NARL well 3

( $\mathrm{TI} 271$ ) showing the A, wellhead and concrete cover of the access pit, and

$B$, sampling port on supply pipe in the pit.

3. Photograph showing equipment for sampling groundwater for radium ...........................14

4. Map showing finite-difference grid and boundary conditions for the groundwater-flow model of Marsh Creek valley, Tioga County, Pennsylvania....

5. Map showing steady-state groundwater budgets for average and dry hydrologic conditions without groundwater withdrawals simulated by the groundwater-flow model of Marsh Creek valley, Tioga County, Pennsylvania 
6. Map showing simulated $A$, areas contributing recharge to wells and water-table contours, B, sources of water to wells, and C, hydraulic response of the aquifer during average hydrologic conditions with withdrawals of 1,000 gallons per minute from the Northern Appalachian Research Laboratory (NARL) wells, Tioga County, Pennsylvania

7. Map showing simulated $A$, areas contributing recharge to wells and water-table contours, $B$, sources of water to wells, and C, hydraulic response of the aquifer during average hydrologic conditions with withdrawals of 2,000 gallons per minute from the Northern Appalachian Research Laboratory (NARL) wells, Tioga County, Pennsylvania

8. Map showing simulated $A$, areas contributing recharge to wells and water-table contours, B, sources of water to wells, and C, hydraulic response of the aquifer during average hydrologic conditions with withdrawals of 2,000 gallons per minute from the Northern Appalachian Research Laboratory (NARL) wells and 1,000 gallons per minute from the new well, Tioga County, Pennsylvania.

9. Map showing simulated additional drawdown caused by a proposed groundwater withdrawal of 1,000 gallons per minute southwest of the Northern Appalachian Research Laboratory (NARL) during average hydrologic conditions, Tioga County, Pennsylvania

10. Map showing simulated $A$, areas contributing recharge to wells and water-table contours, B, sources of water to wells, and C, hydraulic response of the aquifer during average hydrologic conditions with withdrawals of 3,000 gallons per minute from the Northern Appalachian Research Laboratory (NARL) wells and 1,000 gallons per minute from a new production well, Tioga County, Pennsylvania........31

11. Map showing simulated $A$, areas contributing recharge to wells and water-table contours, B, sources of water to wells, and C, hydraulic response of the aquifer during dry hydrologic conditions with withdrawals of 1,000 gallons per minute from the Northern Appalachian Research Laboratory (NARL) wells, Tioga County, Pennsylvania

12. Map showing steady-state simulation of groundwater flow showing $A$, areas contributing recharge to wells and water-level contours, B, sources of water to wells, and $C$, hydraulic response of the aquifer during dry hydrologic conditions with withdrawals of 1,000 gallons per minute from the Northern Appalachian Research Laboratory (NARL) wells and 1,000 gallons per minute from a new production well, Tioga County, Pennsylvania

13. Map showing simulated $A$, areas contributing recharge to wells and water-table contours, $B$, sources of water to wells, and C, hydraulic response of the aquifer during dry hydrologic conditions with withdrawals of 2,000 gallons per minute from the Northern Appalachian Research Laboratory (NARL) wells, Tioga County, Pennsylvania

14. Map showing simulated $A$, areas contributing recharge to wells and water-table contours, B, sources of water to wells, and C, hydraulic response of the aquifer during dry hydrologic conditions with withdrawals of 2,000 gallons per minute from the Northern Appalachian Research Laboratory (NARL) wells and 1,000 gallons per minute from a new production well, Tioga County, Pennsylvania.......36

15. Map showing simulated additional drawdown caused by a proposed groundwater withdrawal of 1,000 gallons per minute southwest of the Northern Appalachian Research Laboratory (NARL) during dry hydrologic conditions, Tioga County, Pennsylvania

16. Map showing simulated areas contributing recharge to wells and extent of the areas captured during 100 or 200 days of steady pumping during dry hydrologic conditions in the model area, Tioga County, Pennsylvania ... 


\section{Tables}

1. Water-quality characteristics and constituents from a periodic sampling program for selected ions and other measures in groundwater from wells sampled in the Marsh Creek valley, Tioga County, Pennsylvania, 2010.

2. Characteristics and identifiers of wells from which samples were collected in the Marsh Creek valley, Tioga County, Pennsylvania, November 2-4, 2010...

3. Water-quality characteristics, major ions, and nutrients in groundwater from wells sampled in the Marsh Creek valley, Tioga County, Pennsylvania, November 2-4, 2010

4. Trace metals and radiochemicals in groundwater from wells sampled in the Marsh Creek valley, Tioga County, Pennsylvania, November 2-4, 2010.

5. Detailed radiochemical analytical results for water samples collected from wells in the Marsh Creek valley, Tioga County, Pennsylvania, November 2-4, 2010.

6. Dissolved gases in groundwater from wells sampled in the Marsh Creek valley, Tioga County, Pennsylvania, November 2-4, 2010.

7. Stable isotopic composition of hydrogen, oxygen, and carbon in groundwater from wells sampled in the Marsh Creek valley, Tioga County, Pennsylvania, November 2-4, 2010

8. Summary of laboratories, laboratory codes, and methods used to analyze water samples collected in the Marsh Creek valley, Tioga County, Pennsylvania, November 2-4, 2010

9. Analytical results for the environmental sample and the sequential replicate of groundwater from well 2 (TI 270) in the Marsh Creek valley, Tioga County, Pennsylvania, November 3, 2010 ...

10. Performance of the U.S. Geological Survey National Water Quality Laboratory in analyzing standard-reference samples in the fall 2010 inter-laboratory comparison study

11. Hydrologic conditions and pumping rates simulated by the steady- state groundwater-flow model of Marsh Creek valley, near Wellsboro, Tioga County, Pennsylvania. 


\section{Conversion Factors, Datum and Abbreviations}

\begin{tabular}{|c|c|c|}
\hline Multiply & By & To obtain \\
\hline \multicolumn{3}{|c|}{ Length } \\
\hline foot $(\mathrm{ft})$ & 0.3048 & meter $(\mathrm{m})$ \\
\hline mile (mi) & 1.609 & kilometer $(\mathrm{km})$ \\
\hline \multicolumn{3}{|c|}{ Area } \\
\hline square mile $\left(\mathrm{mi}^{2}\right)$ & 2.590 & square kilometer $\left(\mathrm{km}^{2}\right)$ \\
\hline \multicolumn{3}{|c|}{ Volume } \\
\hline gallon (gal) & 3.785 & liter (L) \\
\hline liter (L) & 0.2642 & gallon (gal) \\
\hline \multicolumn{3}{|c|}{ Flow Rate } \\
\hline foot per day (ft/d) & 0.3048 & meter per day $(\mathrm{m} / \mathrm{d})$ \\
\hline cubic foot per second ( $\left.\mathrm{ft}^{3} / \mathrm{s}\right)$ & 0.02832 & cubic meter per second $\left(\mathrm{m}^{3} / \mathrm{s}\right)$ \\
\hline gallon per minute (gal/min) & 0.06309 & liter per second $(\mathrm{L} / \mathrm{s})$ \\
\hline \multicolumn{3}{|c|}{ Mass } \\
\hline $\operatorname{gram}(\mathrm{g})$ & 0.03527 & ounce, avoirdupois (oz) \\
\hline \multicolumn{3}{|c|}{ Pressure } \\
\hline inch of mercury at $60^{\circ} \mathrm{F}$ (in $\mathrm{Hg}$ ) & 3.377 & kilopascal (kPa) \\
\hline \multicolumn{3}{|c|}{ Radioactivity } \\
\hline picocurie per liter (pCi/L) & 0.037 & becquerel per liter $(\mathrm{Bq} / \mathrm{L})$ \\
\hline
\end{tabular}

Temperature in degrees Celsius $\left({ }^{\circ} \mathrm{C}\right)$ may be converted to degrees Fahrenheit $\left({ }^{\circ} \mathrm{F}\right)$ as follows:

$$
{ }^{\circ} \mathrm{F}=\left(1.8 x^{\circ} \mathrm{C}\right)+32
$$

Vertical coordinate information is referenced to theNational Geodetic Vertical Datum of 1929 (NGVD 29)."

Horizontal coordinate information is referenced to the North American Datum of 1983 (NAD 83).

Altitude, as used in this report, refers to distance above the vertical datum.

Specific conductance is given in microsiemens per centimeter at 25 degrees Celsius $\left(\mu \mathrm{S} / \mathrm{cm}\right.$ at $\left.25^{\circ} \mathrm{C}\right)$.

Concentrations of chemical constituents in water are given either in milligrams per liter (mg/L) or micrograms per liter ( $\mu \mathrm{g} / \mathrm{L})$ or picocuries per liter ( $\mathrm{pC} \mathrm{C} / \mathrm{L})$. 


\section{List of Acronyms}

ARGUS Argus Numerical Environments

MCL maximum contaminant level

NARL Northern Appalachian Research Laboratory

NWOL National Water Quality Laboratory

PADEP Pennsylvania Department of Environmental Protection

SRBC Susquehanna River Basin Commission

USEPA U.S. Environmental Protection Agency

USGS U.S. Geological Survey 
This page has been left blank intentionally. 


\title{
Ground water Quality and Simulation of Sources of Water to Wells in the Marsh Creek Valley at the U.S. Geological Survey Northern Appalachian Research Laboratory, Tioga County, Pennsylvania
}

\author{
By Dennis W. Risser and Kevin J. Breen
}

\section{Abstract}

This report provides a November 2010 snapshot of groundwater quality and an analysis of the sources of water to wells at the U.S. Geological Survey (USGS) Northern Appalachian Research Laboratory (NARL) near Wellsboro, Pennsylvania. The laboratory, which conducts fisheries research, currently (2011) withdraws 1,000 gallons per minute of high-quality groundwater from three wells completed in the glacial sand and gravel aquifer beneath the Marsh Creek valley; a fourth well that taps the same aquifer provides the potable supply for the facility. The study was conducted to document the source areas and quality of the water supply for this Department of Interior facility, which is surrounded by the ongoing development of natural gas from the Marcellus Shale.

Groundwater samples were collected from the four wells used by the NARL and from two nearby domestic-supply wells. The domestic-supply wells withdraw groundwater from bedrock of the Catskill Formation. Samples were analyzed for major ions, nutrients, trace metals, radiochemicals, dissolved gases, and stable isotopes of oxygen and hydrogen in water and carbon in dissolved carbonate to document groundwater quality. Organic constituents (other than hydrocarbon gases) associated with hydraulic fracturing and other human activities were not analyzed as part of this assessment. Results show low concentrations of all constituents. Only radon, which ranged from 980 to 1,310 picocuries per liter, was somewhat elevated. These findings are consistent with the pristine nature of the aquifer in the Marsh Creek valley, which is the reason the laboratory was sited at this location.

The sources of water and areas contributing recharge to wells were identified by the use of a previously documented MODFLOW groundwater-flow model for the following conditions: (1) withdrawals of 1,000 to 3,000 gallons per minute from the NARL wells, (2) average or dry hydrologic conditions, and (3) withdrawals of 1,000 gallons per minute from a new well 3,500 feet to the southwest that was drilled to provide water for Marcellus gas-well operations. Results of simulations indicate that during average hydrologic conditions, infiltration from Straight Run, a tributary to Marsh Creek, provides nearly all the water to the NARL wells. During dry conditions, the areas contributing recharge expand such that Asaph Run contributes about half of the water to the NARL wells when withdrawals are 1,000 or 2,000 gallons per minute. The addition of a simulated withdrawal of 1,000 gallons per minute from the nearby new well does not substantially affect the sources of water captured by the NARL wells.

These results are subject to some limitations. The waterquality samples represent a snapshot of groundwater chemistry for only one hydrologic condition; the concentrations of some constituents may change temporally. In addition, samples were collected and analyzed for hydrocarbon gases, but not organic constituents associated with hydraulic fracturing; additional sampling for these constituents would provide a more complete water-quality baseline. The sources contributing water to the NARL wells and the new well were simulated by use of a simplified one-layer model of the glacial sand and gravel aquifer for steady-state conditions that in reality are never achieved. Steady-state simulations of dry hydrologic conditions show that it is possible for the NARL wells to capture water from Asaph Run; however, maps of simulated groundwater time-of-travel indicate that a dry period of unusually long duration would be required. A better analysis could be done by recalibrating the groundwater-flow model with a finite-difference grid having multiple layers, cells smaller than the 200 -foot by 200 -foot cells used in this study, and transient stress periods.

\section{Introduction}

Development of natural-gas resources from the Marcellus Shale is underway in the vicinity of the U.S. Geological Survey (USGS) Northern Appalachian Research Laboratory (NARL) near Wellsboro, Pennsylvania (fig. 1). Gas wells are being permitted and drilled in the Marsh Creek valley and adjacent uplands to the north and south of the valley. From November 2009 to June 2010, 50 wells 
were permitted within a 200 -square-mile area $\left(\mathrm{mi}^{2}\right)$ around the NARL. Land disturbance from access roads, gas wells, and pipeline construction is anticipated, and hydrologic effects from hydraulic fracturing with associated water use and waste handling are possible. A monitoring program is needed to document potential effects of the gas exploration and production activities on the quantity and quality of water produced by wells completed in and adjacent to the valley.

The NARL is an aquatic biology research station of the USGS with facilities that include an outdoor raceway system and wet and dry laboratories. The NARL was established in the late 1970s after a lengthy search for a pristine water supply of sufficient quantity and quality. Water for operations of the 55-acre, four-building complex is obtained from four wells that tap the glacial sand and gravel aquifer beneath the Marsh Creek valley.

Wells 1,3 , and 5 withdraw a combined total of 1,000 gallons per minute ( $\mathrm{gal} / \mathrm{min}$ ) for fisheries research, and well 2 provides the potable supply for the facility. Hereafter, the four wells are collectively termed the NARL wells. The NARL groundwater withdrawals are nearly 100 percent non-consumptive. The small withdrawals for domestic-supply use from well 2 are returned through an on-site wastewater leach field, and the withdrawals for the fish hatchery from wells 1,3 , and 5 are discharged about 400 feet (ft) downstream from the confluence of Straight Run and Marsh Creek. The metered discharge is equal to or greater than the metered total withdrawals from the wells (William Lellis, Director, U.S. Geological Survey Northern Appalachian Research Laboratory, oral commun., 2011). Total groundwater withdrawals of as much as $3,000 \mathrm{gal} / \mathrm{min}$ are permitted by the Susquehanna River Basin Commission under certain hydrologic conditions provided that the streamflow in Straight Run and groundwater levels beneath wetlands are monitored (Susquehanna River Basin Commission, 2003).

The NARL is a public resource that is used to perform large-scale laboratory and field research on aquatic organisms. To help protect Department of Interior interests and infrastructure from the possible effects of gas exploration and production activities, a study was initiated on October 1, 2010, to collect initial samples to document groundwater quality in the glacial sand and gravel and the bedrock aquifers, and to identify the sources of water for the NARL wells.

The glacial sand and gravel aquifer in the Marsh Creek valley near the NARL is a potential source of water for hydraulic fracturing operations associated with gas-well development. A permit to withdraw $1,000 \mathrm{gal} / \mathrm{min}$ of water from a new well in the glacial sand and gravel aquifer $3,500 \mathrm{ft}$ southwest of the NARL is now (March 2011) pending with the Susquehanna River Basin Commission (SRBC). Use of water for hydraulic fracturing is assumed to be 100 percent consumptive by the SRBC, so in this study, simulated withdrawals of water from this well are assumed to be lost to the glacial sand and gravel aquifer. A determination of the areas contributing recharge and sources of water to NARL wells is needed to protect the water supply from potential sources of contamination and possible declines in yield as a result of additional withdrawals. Understanding the sources of water and potential flow paths to the NARL wells is important if the need arises to modify operations to address the transport of contaminants that may enter the glacial sand and gravel aquifer from a source related to resource extraction or other activities.

The glacial sand and gravel aquifer is composed of interbedded silty sand and gravel lenses, laid down as icecontact and stream deposits, overlain by alluvium and finegrained swamp deposits (Williams, 1991). In the vicinity of the NARL, the glacial sand and gravel aquifer ranges from a few feet thick at the bounding valley walls to a maximum of about $120 \mathrm{ft}$. The glaciated valley and surrounding uplands are underlain mostly by sandstone and shale of Devonian through Pennsylvanian age, mantled by less than $10 \mathrm{ft}$ of till. The bedrock is a much less productive aquifer than the glacial-drift deposits beneath the valley floor. Williams (1991) determined that recharge to the glacial sand and gravel aquifer is derived mostly from infiltration of water from Straight Run and Asaph Run (71 percent of the total). The remainder of the recharge is provided by unchanneled runoff, groundwater inflow from upvalley, and precipitation on the valley.

\section{Purpose and Scope}

This report provides (1) a snapshot during November $2-4,2010$, of the groundwater quality in the four NARL wells completed in the glacial sand and gravel aquifer and in two nearby private domestic-supply wells completed in fractured bedrock and (2) an estimation of the areas contributing recharge and sources of water to wells at the NARL by the use of a previously documented groundwater-flow model of the glacial sand and gravel aquifer. Groundwater-quality data include concentrations of major ions, nutrients, trace elements, radiochemicals, dissolved gases, and stable isotopes of oxygen and hydrogen in water and carbon in dissolved carbonate; these data provide some documentation of the groundwater geochemistry of the glacial sand and gravel and bedrock aquifers at and near the NARL. This study does not establish a baseline for organic constituents (other than hydrocarbon gases) associated with hydraulic fracturing activities, though establishing a baseline for selected anthropogenic organic compounds would provide a more complete water-quality baseline and would be a useful objective for future sampling. The areas contributing recharge and sources of groundwater to wells were determined from simulations of steady-state groundwater flow conducted by the use of a two-dimensional MODFLOW model previously documented by Williams and Morrissey (1996).

\section{Previous Investigations}

Williams (1991) describes the hydrology of the Marsh Creek valley and the hydraulics of groundwater/surface-water 

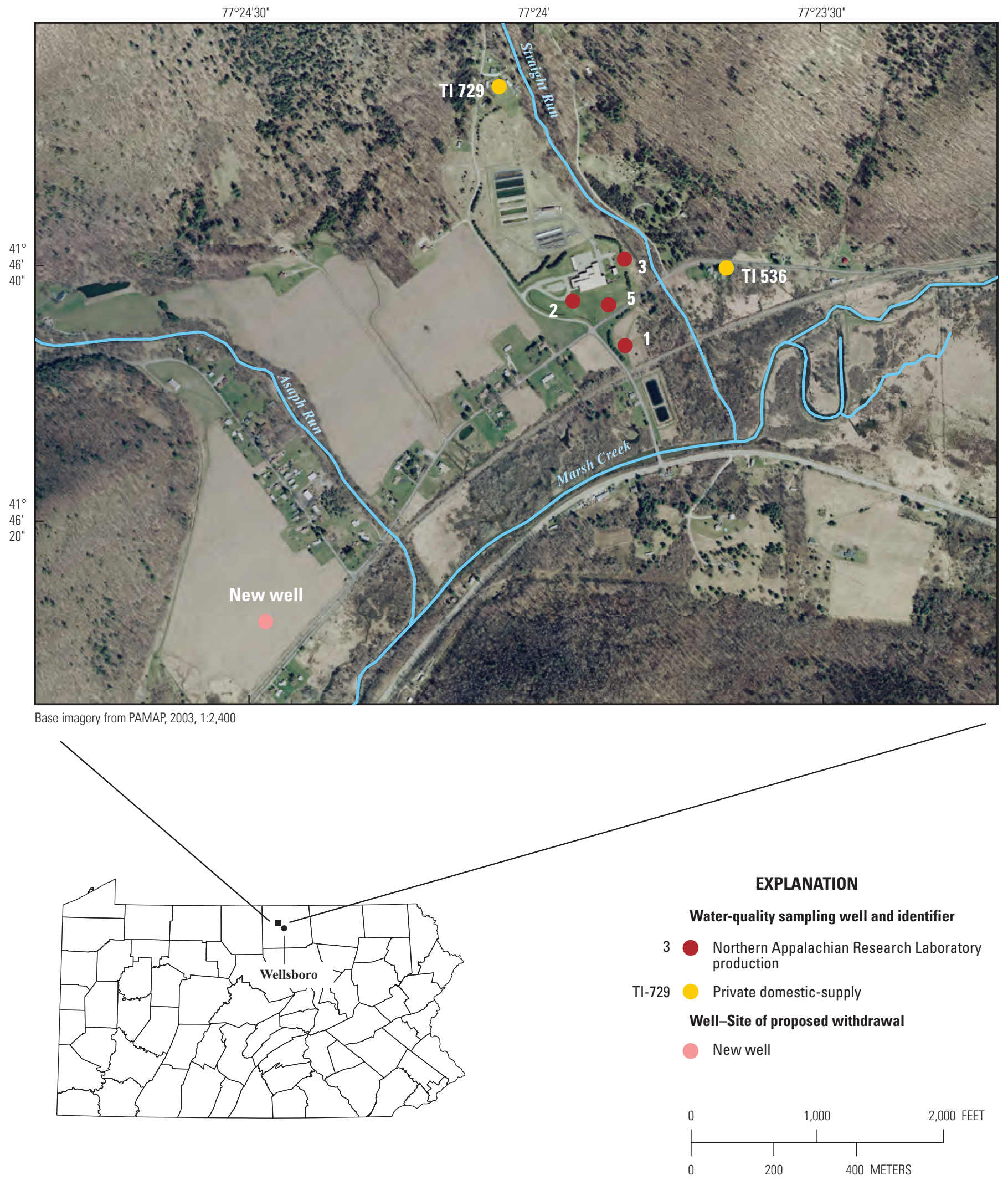

Figure 1. Location of the study area in the Marsh Creek valley, near Wellsboro, Tioga County, Pennsylvania. 
interactions at the NARL (formerly the National Fisheries Research and Development Laboratory). Williams established that infiltration from streams draining the bedrock uplands provided 71 percent of the recharge to the glacial sand and gravel aquifer and described the concentrations of selected major ions in groundwater. Risser and Madden (1994) used a two-dimensional groundwater model to compare different approaches for delineating areas contributing recharge to the NARL wells, but this (1992) model was not calibrated to observations of groundwater levels or streamflow; thus, those results should be considered rough estimates. Williams and Morrissey (1996) constructed a calibrated two-dimensional groundwater model to simulate stream seepage and generate water budgets for the glacial sand and gravel aquifer but did not delineate areas contributing recharge to the NARL wells.

Water-quality samples from the NARL wells were collected and analyzed during the spring (February and March) and fall (October and November) of 2010 by a private laboratory. The samples were analyzed for major ions, trace metals, bacteria, and methane to provide background information on constituents that are often associated with contamination from the extraction of natural gas. Results from the private laboratory are listed in table 1 to allow for comparison to the suite of constituents analyzed at lower detection limits for this study.

\section{Study Methods}

The study was conducted by sampling groundwater from wells and simulating groundwater flow by use of a numerical model. Groundwater samples were analyzed and quality-assurance measures were reported. Simulations of groundwater flow were accomplished by updating an available numerical model and simulating the sources of water and areas contributing recharge to wells.

\section{Groundwater-Quality Sampling}

Groundwater samples were collected from the four NARL wells and two nearby privately owned domesticsupply wells during November 2-4, 2010. The locations of the wells are shown in figure 1, and the local names and USGS identifiers are cross referenced in table 2. Samples were analyzed for broad sets of chemical constituents at detection levels that allow concentrations at low levels to be quantified. Organic constituents associated with hydraulicfracturing activities were not analyzed, primarily because of budget limitations.

\section{Collection and Analysis of Samples}

Water samples were collected from the wells and were processed using standard field methods of the USGS (U.S. Geological Survey, variously dated). The NARL wells had been pumping for at least 24 hours prior to sampling, and the private domestic-supply wells were purged using the homeowner's pump, then sampled after stabilization of field water-quality characteristics. Water was collected from the wells through a 15 -ft length of polytetrafluoroethylene(PTFE) lined plastic tubing, hereafter termed "sampling tubing," with a 0.45 -micron capsule filter attached to the discharge end of the tubing, which minimized exposure of the sample to the atmosphere. Water samples were obtained directly from wells 1,3 , and 5 (TI 269, TI 271, and TI 730, respectively) by attaching the sampling tubing to the sampling port on the distribution pipe that was accessible in a pit adjacent to the well (fig. 2). Water from well 2 (TI 270) was obtained through the sampling tubing attached to a spigot on a large (about 1,200 gal) holding tank in the laboratory isolation room. The spigot on the tank was opened for 24 hours prior to sampling to flush out the water stored in the tank. Water from the private domestic-supply wells was collected through the sampling tubing attached to a spigot on the pressure tank inside the house at well TI 729 and attached to the frost-free hydrant at the wellhead for well TI 536.

Water from each well was analyzed for field properties, major ions, nutrients, trace metals, radiochemicals, dissolved gases, and stable isotopes (tables 3-7). The $\mathrm{pH}$, water temperature, specific conductance, dissolved oxygen, and oxidation-reduction potential were measured in the field with a multi-parameter meter and flow-through chamber that was connected to the sampling tubing. Alkalinity (acid neutralizing capacity) was titrated in the field at the time of sample collection. Samples were processed according to protocols outlined in Wilde and others (2004) and, except for the samples for radium and uranium, were preserved and chilled (if necessary), then shipped to the appropriate laboratory by overnight express under chain-of-custody protocols. Radium and uranium isotopes were processed in the field by flushing at least 100 gallons of water directly from the well though filters impregnated with potassium permanganate (fig. 3). Those filters were driven directly to the USGS radiochemistry laboratory in Reston, Virginia, for analysis after all wells were sampled.

The following laboratories were used to analyze the water samples. Details about the laboratory codes and methods for each constituent are listed in table 8 .

- USGS National Water Quality Laboratory (NWQL) Major ions, nutrients, trace metals, and radon.

- Eberline Services, Inc.-Gross alpha and gross beta radioactivity.

- USGS radiochemical laboratory in Reston, VirginiaIsotopes of radium and uranium.

- Isotech Laboratories, Inc.-Dissolved gases and stable isotopes.

The NWQL is certified by the National Environmental Laboratory Accreditation Program for analysis of nonpotable waters using USGS methods. Details about the NWQL 


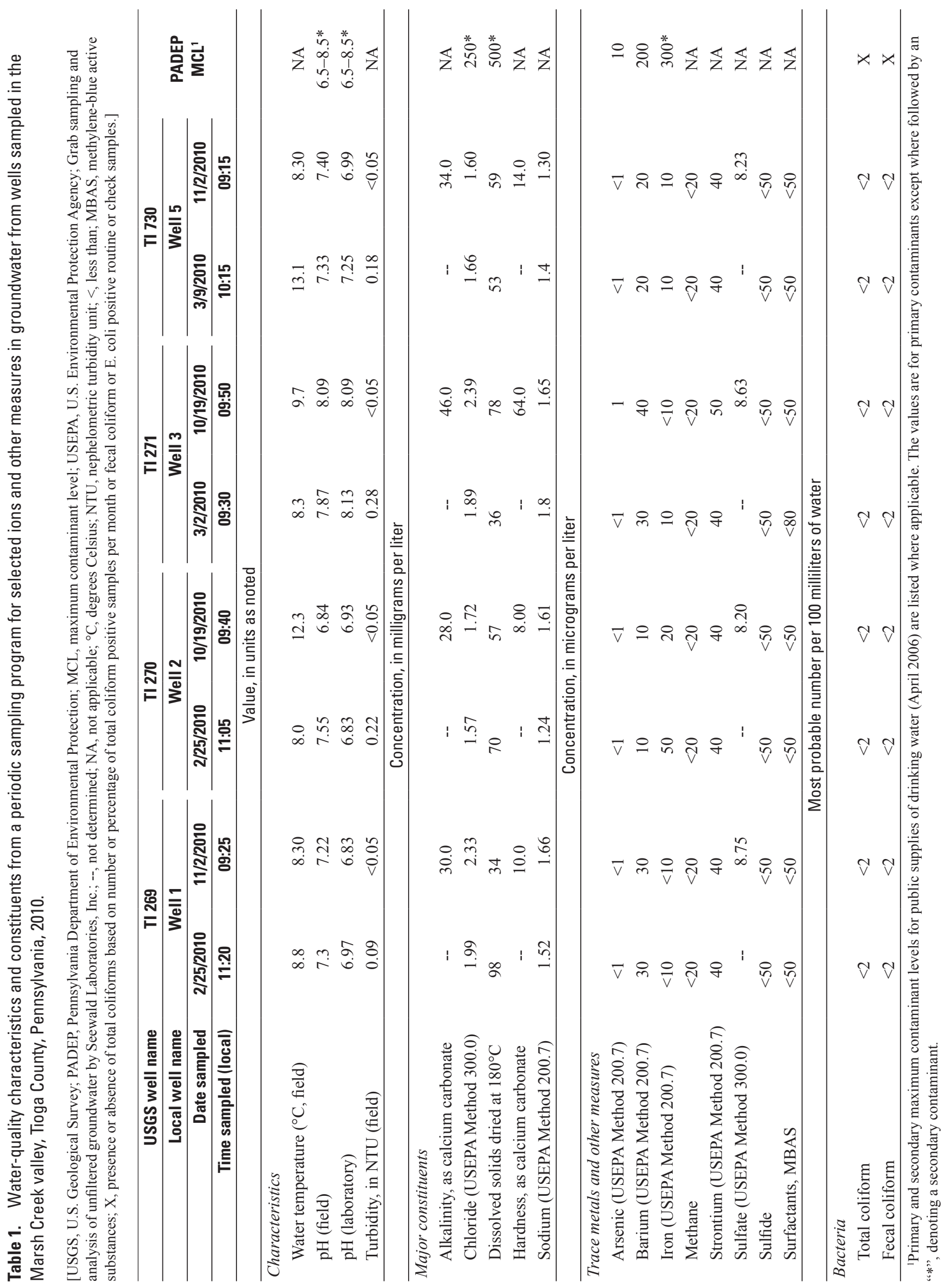




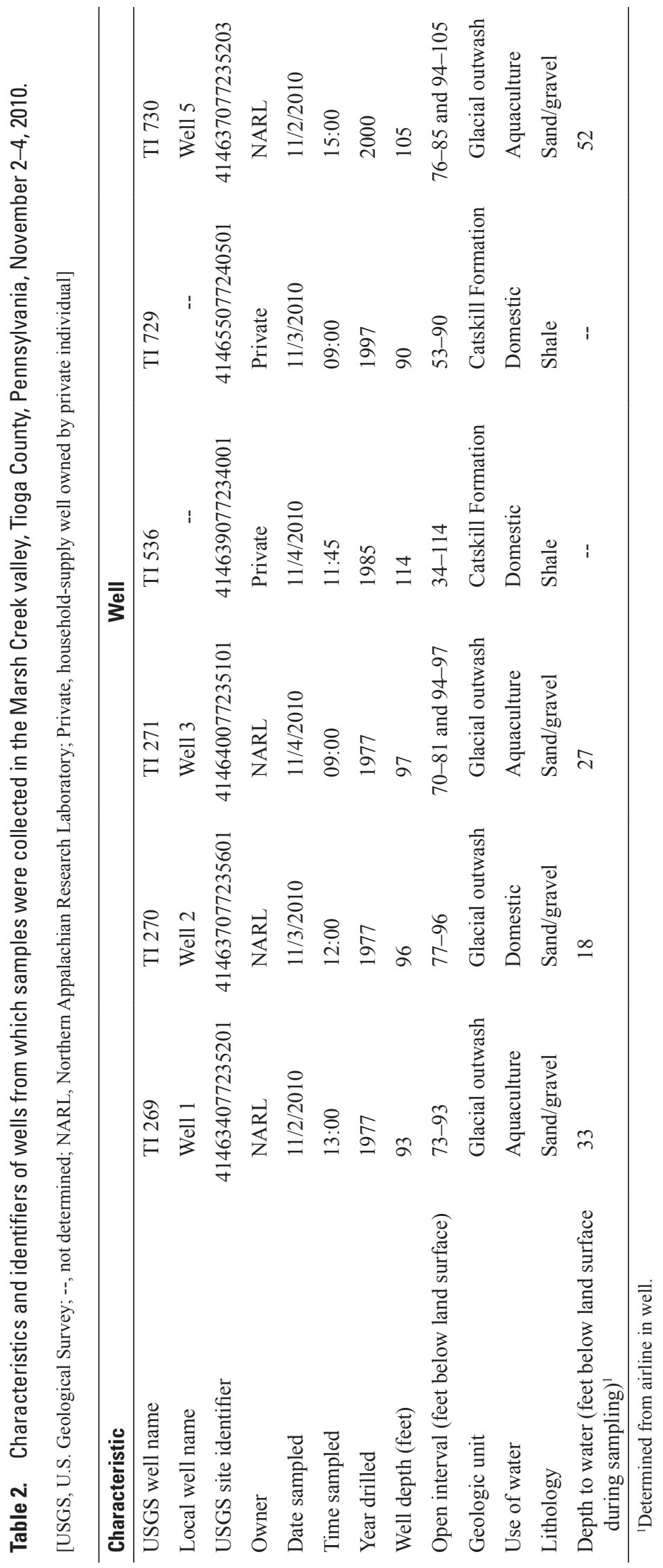



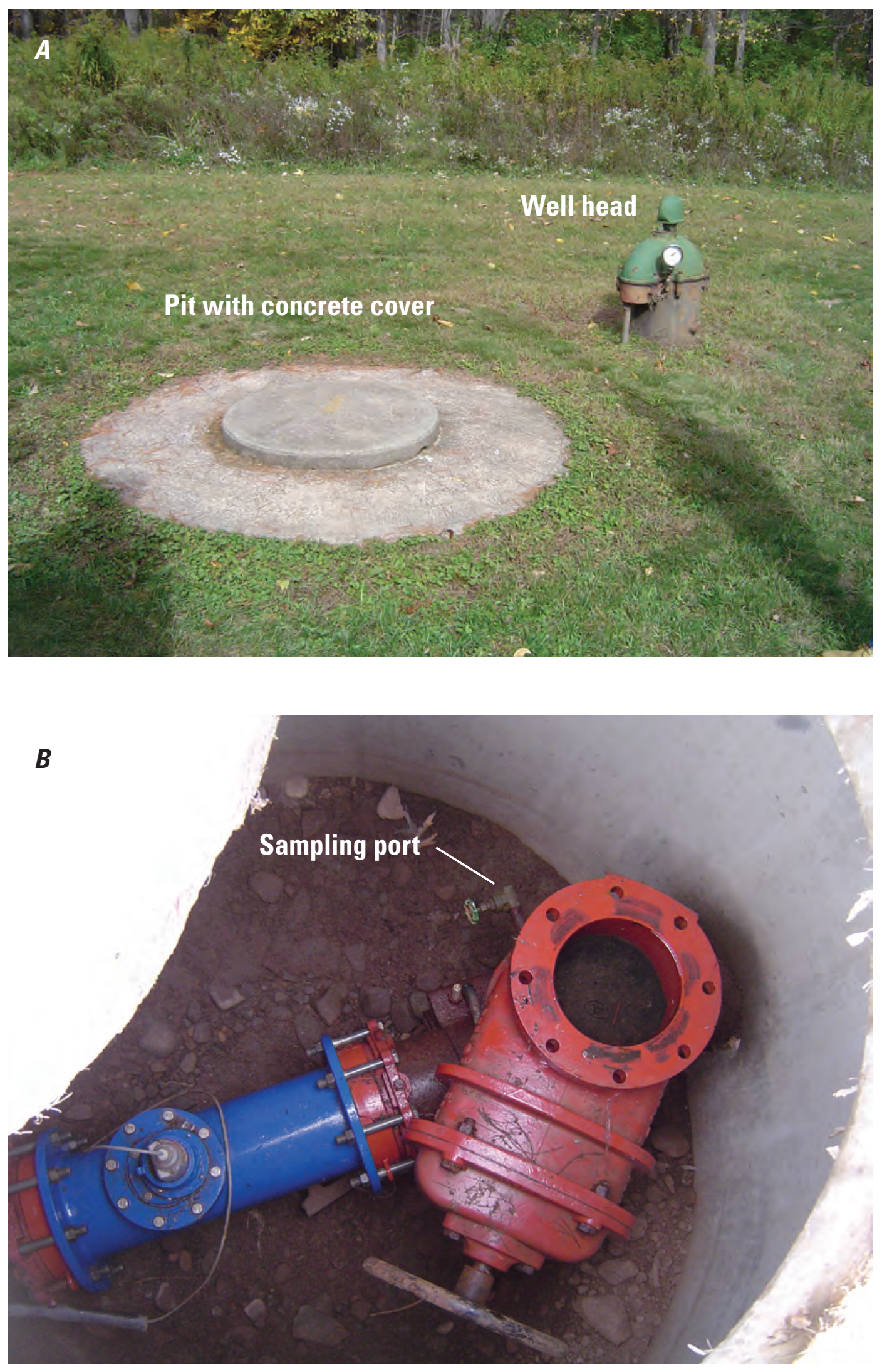

Figure 2. Northern Appalachian Research Laboratory NARL well 3 (TI 271) showing the $A$, wellhead and concrete cover of the access pit, and $B$, sampling port on supply pipe in the pit. 


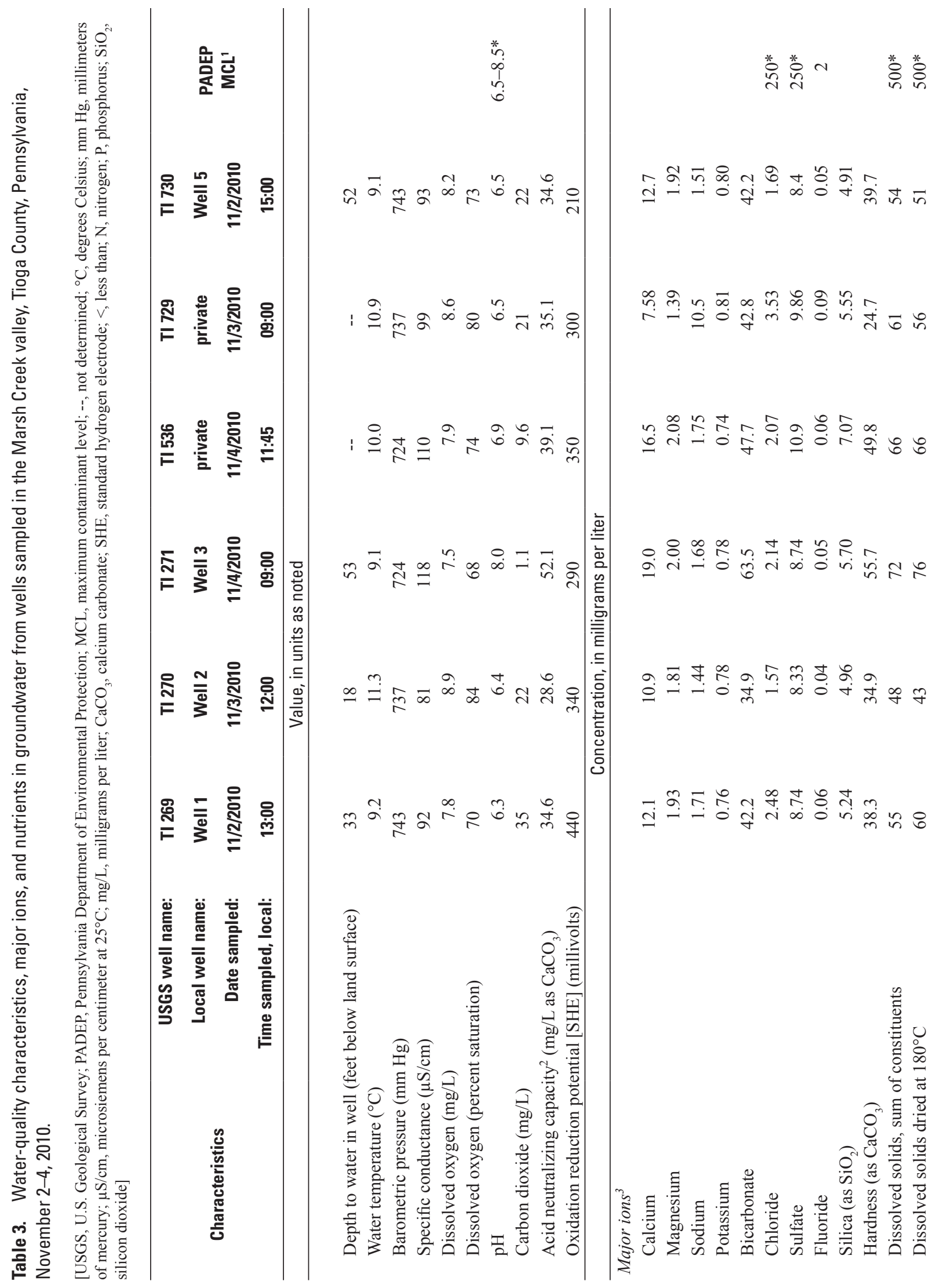




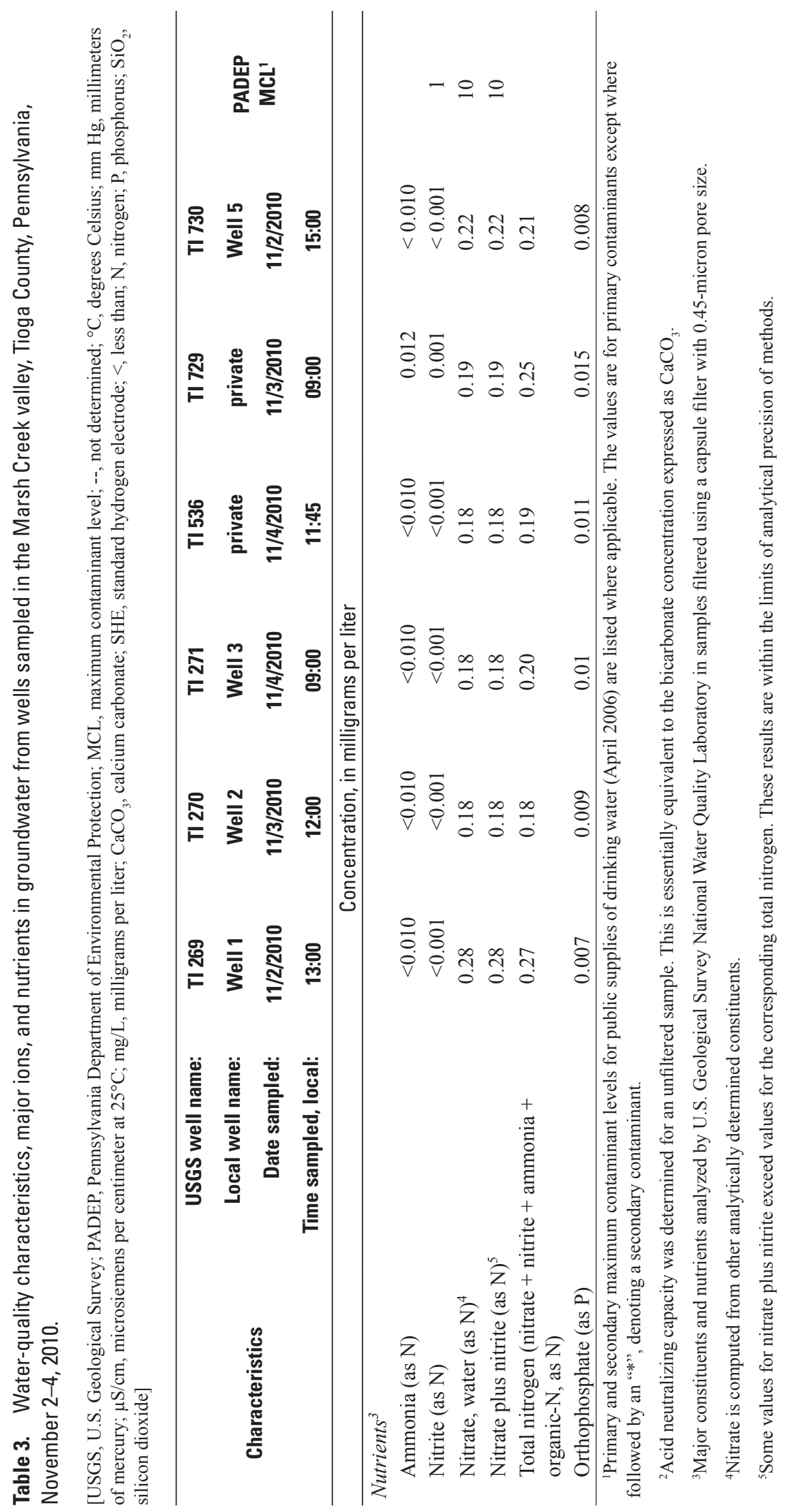


Table 4. Trace metals and radiochemicals in groundwater from wells sampled in the Marsh Creek valley, Tioga County, Pennsylvania, November 2-4, 2010.

[USGS; U.S. Geological Survey; PADEP; Pennsylvania Department of Environmental Protection; MCL, maximum contaminant level. Samples were filtered through a membrane with 0.45 -micron pore size for all analyses except radon; $<$, less than; ND, not detected indicates results for radiochemicals detected at concentrations less than the site-specific critical level ( $\mathrm{ssL}_{\mathrm{c}}$ ) when the $\mathrm{ssL}_{\mathrm{c}}$ was negative; $72-\mathrm{h}$, sample analyzed for concentration at approximately 72 hours after sample collection as referenced to a detector calibrated using ${ }^{230} \mathrm{Th}$ for gross alpha and ${ }^{137} \mathrm{Cs}$ for gross beta; 30 -d, sample used for the 72 -hour analysis is counted a second time approximately 30 days after the initial count as referenced to a detector calibrated using ${ }^{230} \mathrm{Th}$ for gross alpha and ${ }^{137} \mathrm{Cs}$ for gross beta]

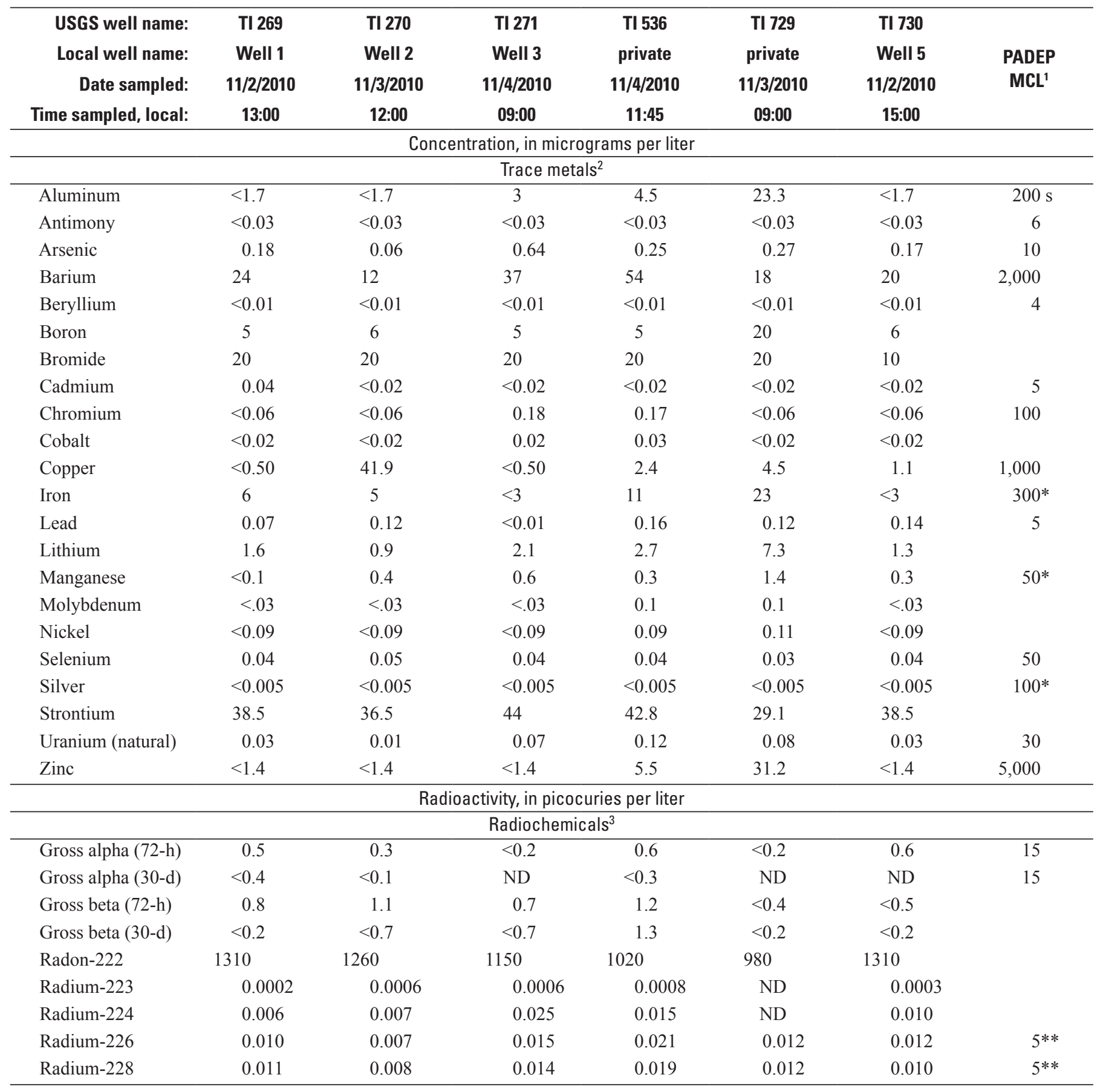

${ }^{1}$ Primary and secondary maximum contaminant levels for public supplies of drinking water (April 2006) are listed where applicable. The values are for primary contaminants except where followed by an “*”, denoting a secondary contaminant. The lead and copper primary MCLs are applicable only to bottled, vended, retail, and bulk water hauling systems. “**” denotes that the MCL for radium is for radium-226 plus radium 228.

${ }^{2}$ Trace metals analyzed by U.S. Geological Survey National Water Quality Laboratory.

${ }^{3}$ Radon-222 analyzed by U.S. Geological Survey National Water Quality Laboratory. Radium-223, 224, 226, and 228 analyzed by USGS Reston Radiochemical Laboratory. Gross alpha and beta analyzed by Eberline Services, Inc. 
Table 5. Detailed radiochemical analytical results for water samples collected from wells in the Marsh Creek valley, Tioga County, Pennsylvania, November 2-4, 2010.

[Result, radiological concentration; CSU, combined standard uncertainty (1-sigma); $\mathrm{ssL}_{\mathrm{c}}$, sample-specific critical level; 72-h, sample analyzed for concentration at approximately 72 hours after sample collection as referenced to a detector calibrated using ${ }^{230} \mathrm{Th}$ for gross alpha and ${ }^{137} \mathrm{Cs}$ for gross beta; 30-d, sample used for the 72-hour analysis is counted a second time approximately 30 days after the initial count as referenced to a detector calibrated using ${ }^{230} \mathrm{Th}$ for gross alpha and ${ }^{137} \mathrm{Cs}$ for gross beta; $\mathrm{pCi} / \mathrm{L}$; picocuries per liter; $\mathrm{D}$, analyte detected; ND, analyte not detected, concentration is less than the sample-specific critical level; a, blank is greater than the $\mathrm{ssL}_{\mathrm{c}}$; b, value extrapolated at low end; z, sample-specific critial level not reported by laboratory; EBER, Eberline Services, Inc.; NWQL, U.S. Geological Survey National Water Quality Laboratory; REST, U.S. Geological Survey radiochemical laboratory in Reston, Virginia]

\begin{tabular}{|c|c|c|c|c|c|c|c|}
\hline $\begin{array}{c}\text { Well } \\
\text { identifier }\end{array}$ & $\begin{array}{c}\text { Sample } \\
\text { collection } \\
\text { date and time }\end{array}$ & $\begin{array}{c}\text { Radiological } \\
\text { constituent }\end{array}$ & $\begin{array}{l}\text { Result } \\
\text { (pCi/L) }\end{array}$ & $\underset{(p C i / L)}{C S U}$ & Remarks & $\begin{array}{c}s s L_{c} \\
(p C i / L)\end{array}$ & Laboratory \\
\hline \multirow[t]{9}{*}{ TI 269 (Well 1) } & \multirow[t]{9}{*}{ 11/2/10 13:00 } & Gross alpha (72-h) & 0.53 & 0.21 & $\mathrm{D}$ & 0.24 & EBER \\
\hline & & Gross alpha (30-d) & 0.42 & 0.49 & $\mathrm{ND}, \mathrm{a}$ & 0.69 & EBER \\
\hline & & Gross beta (72-h) & 0.78 & 0.27 & $\mathrm{D}$ & 0.41 & EBER \\
\hline & & Gross beta $(30-d)$ & 0.2 & 0.49 & ND & 0.78 & EBER \\
\hline & & Radon-222 & 1,310 & 74 & $\mathrm{D}$ & 11.8 & NWQL \\
\hline & & Radium-223 & 0.0002 & 0.00004 & $\mathrm{D}$ & $\mathrm{z}$ & REST \\
\hline & & Radium-224 & 0.006 & 0.0003 & $\mathrm{D}$ & $\mathrm{z}$ & REST \\
\hline & & Radium-226 & 0.010 & 0.0005 & $\mathrm{D}$ & $\mathrm{z}$ & REST \\
\hline & & Radium-228 & 0.011 & 0.0004 & $\mathrm{D}$ & $\mathrm{z}$ & REST \\
\hline \multirow[t]{9}{*}{ TI 270 (Well 2) } & \multirow[t]{9}{*}{$11 / 3 / 1012: 00$} & Gross alpha (72-h) & 0.35 & 0.23 & $\mathrm{D}$ & 0.26 & EBER \\
\hline & & Gross alpha (30-d) & 0.12 & 0.28 & $\mathrm{ND}, \mathrm{a}$ & 0.4 & EBER \\
\hline & & Gross beta (72-h) & 1.11 & 0.39 & $\mathrm{D}$ & 0.58 & EBER \\
\hline & & Gross beta (30-d) & 0.7 & 0.75 & ND & 1.3 & EBER \\
\hline & & Radon-222 & 1,260 & 71 & $\mathrm{D}$ & 12.7 & NWQL \\
\hline & & Radium-223 & 0.0006 & 0.00014 & $\mathrm{D}$ & $\mathrm{z}$ & REST \\
\hline & & Radium-224 & 0.007 & 0.0003 & $\mathrm{D}$ & $\mathrm{z}$ & REST \\
\hline & & Radium-226 & 0.007 & 0.0004 & $\mathrm{D}$ & $\mathrm{z}$ & REST \\
\hline & & Radium-228 & 0.008 & 0.0005 & $\mathrm{D}$ & $\mathrm{z}$ & REST \\
\hline \multirow{9}{*}{$\begin{array}{l}\text { TI } 270 \text { (Well 2) } \\
\text { replicate }\end{array}$} & \multirow[t]{9}{*}{ 11/3/10 12:01 } & Gross alpha (72-h) & 0.3 & 0.26 & ND & 0.34 & EBER \\
\hline & & Gross alpha (30-d) & -0.09 & 0.33 & $\mathrm{ND}, \mathrm{a}, \mathrm{b}$ & 0.56 & EBER \\
\hline & & Gross beta (72-h) & -0.17 & 0.44 & $\mathrm{ND}, \mathrm{b}$ & 0.72 & EBER \\
\hline & & Gross beta (30-d) & 0.3 & 0.5 & ND & 0.83 & EBER \\
\hline & & Radon-222 & 1,244 & 71 & $\mathrm{D}$ & 12.9 & NWQL \\
\hline & & Radium-223 & 0.0006 & 0.00014 & $\mathrm{D}$ & $\mathrm{z}$ & REST \\
\hline & & Radium-224 & 0.007 & 0.0003 & $\mathrm{D}$ & $\mathrm{z}$ & REST \\
\hline & & Radium-226 & 0.007 & 0.0004 & $\mathrm{D}$ & $\mathrm{z}$ & REST \\
\hline & & Radium-228 & 0.008 & 0.0005 & $\mathrm{D}$ & $\mathrm{z}$ & REST \\
\hline \multirow[t]{9}{*}{ TI 271 (Well 3) } & \multirow[t]{9}{*}{ 11/4/10 9:00 } & Gross alpha (72-h) & 0.18 & 0.27 & ND & 0.37 & EBER \\
\hline & & Gross alpha (30-d) & -0.38 & 0.31 & $\mathrm{ND}, \mathrm{b}$ & 0.6 & EBER \\
\hline & & Gross beta (72-h) & 0.73 & 0.38 & $\mathrm{D}$ & 0.57 & EBER \\
\hline & & Gross beta $(30-d)$ & 0.7 & 0.5 & ND & 0.81 & EBER \\
\hline & & Radon-222 & 1,150 & 65 & $\mathrm{D}$ & 13.2 & NWQL \\
\hline & & Radium-223 & 0.0006 & 0.00006 & $\mathrm{D}$ & $\mathrm{z}$ & REST \\
\hline & & Radium-224 & 0.025 & 0.0005 & $\mathrm{D}$ & $\mathrm{z}$ & REST \\
\hline & & Radium-226 & 0.015 & 0.0004 & $\mathrm{D}$ & $\mathrm{z}$ & REST \\
\hline & & Radium-228 & 0.014 & 0.0006 & $\mathrm{D}$ & $\mathrm{z}$ & REST \\
\hline
\end{tabular}


Table 5. Detailed radiochemical analytical results for water samples collected from wells in the Marsh Creek valley, Tioga County, Pennsylvania, November 2-4, 2010.

[Result, radiological concentration; CSU, combined standard uncertainty (1-sigma); $\mathrm{ssL}_{\mathrm{c}}$, sample-specific critical level; 72-h, sample analyzed for concentration at approximately 72 hours after sample collection as referenced to a detector calibrated using ${ }^{230} \mathrm{Th}$ for gross alpha and ${ }^{137} \mathrm{Cs}$ for gross beta; 30-d, sample used for the 72-hour analysis is counted a second time approximately 30 days after the initial count as referenced to a detector calibrated using ${ }^{230} \mathrm{Th}$ for gross alpha and ${ }^{137} \mathrm{Cs}$ for gross beta; $\mathrm{pCi} / \mathrm{L}$; picocuries per liter; $\mathrm{D}$, analyte detected; $\mathrm{ND}$, analyte not detected, concentration is less than the sample-specific critical level; a, blank is greater than the $\mathrm{ssL}_{\mathrm{c}}$; b, value extrapolated at low end; $\mathrm{z}$, sample-specific critial level not reported by laboratory; EBER, Eberline Services, Inc.; NWQL, U.S. Geological Survey National Water Quality Laboratory; REST, U.S. Geological Survey radiochemical laboratory in Reston, Virginia]

\begin{tabular}{|c|c|c|c|c|c|c|c|}
\hline $\begin{array}{c}\text { Well } \\
\text { identifier }\end{array}$ & $\begin{array}{c}\text { Sample } \\
\text { collection } \\
\text { date and time }\end{array}$ & $\begin{array}{l}\text { Radiological } \\
\text { constituent }\end{array}$ & $\begin{array}{l}\text { Result } \\
\text { (pCi/L) }\end{array}$ & $\underset{(\mathrm{pCi} / \mathrm{L})}{\mathrm{CSU}}$ & Remarks & $\begin{array}{c}s s L_{\mathrm{c}} \\
(\mathrm{pCi} / \mathrm{L})\end{array}$ & Laboratory \\
\hline \multirow[t]{9}{*}{ TI 536} & \multirow[t]{9}{*}{$11 / 4 / 1011: 45$} & Gross alpha (72-h) & 0.6 & 0.3 & $\mathrm{D}$ & 0.3 & EBER \\
\hline & & Gross alpha (30-d) & 0.3 & 0.4 & ND & 0.52 & EBER \\
\hline & & Gross beta (72-h) & 1.2 & 0.4 & $\mathrm{D}$ & 0.59 & EBER \\
\hline & & Gross beta $(30-d)$ & 1.26 & 0.65 & $\mathrm{D}$ & 1 & EBER \\
\hline & & Radon-222 & 1,020 & 59 & $\mathrm{D}$ & 13.1 & NWQL \\
\hline & & Radium-223 & 0.0008 & 0.00005 & $\mathrm{D}$ & $\mathrm{z}$ & REST \\
\hline & & Radium-224 & 0.015 & 0.0003 & $\mathrm{D}$ & $\mathrm{z}$ & REST \\
\hline & & Radium-226 & 0.021 & 0.0006 & $\mathrm{D}$ & $\mathrm{z}$ & REST \\
\hline & & Radium-228 & 0.019 & 0.0011 & $\mathrm{D}$ & $\mathrm{z}$ & REST \\
\hline \multirow[t]{9}{*}{ TI 729} & \multirow[t]{9}{*}{ 11/3/10 9:00 } & Gross alpha (72-h) & 0.2 & 0.3 & ND & 0.44 & EBER \\
\hline & & Gross alpha (30-d) & -0.41 & 0.33 & ND & 0.65 & EBER \\
\hline & & Gross beta (72-h) & 0.43 & 0.37 & ND & 0.58 & EBER \\
\hline & & Gross beta $(30-d)$ & 0.2 & 0.8 & ND & 1.3 & EBER \\
\hline & & Radon-222 & 980 & 57 & $\mathrm{D}$ & 12.8 & NWQL \\
\hline & & Radium-223 & 0.0000 & 0.00000 & ND & $\mathrm{z}$ & REST \\
\hline & & Radium-224 & -0.000 & -0.0000 & ND & $\mathrm{z}$ & REST \\
\hline & & Radium-226 & 0.012 & 0.0004 & $\mathrm{D}$ & $\mathrm{z}$ & REST \\
\hline & & Radium-228 & 0.012 & 0.0005 & $\mathrm{D}$ & $\mathrm{z}$ & REST \\
\hline \multirow[t]{9}{*}{ TI 730 (Well 5) } & \multirow[t]{9}{*}{$11 / 2 / 1015: 00$} & Gross alpha (72-h) & 0.55 & 0.22 & $\mathrm{D}$ & 0.25 & EBER \\
\hline & & Gross alpha (30-d) & -0.23 & 0.21 & ND & 0.39 & EBER \\
\hline & & Gross beta (72-h) & 0.46 & 0.32 & ND & 0.51 & EBER \\
\hline & & Gross beta (30-d) & 0.16 & 0.39 & ND & 0.63 & EBER \\
\hline & & Radon-222 & 1,310 & 74 & $\mathrm{D}$ & 11.7 & NWQL \\
\hline & & Radium-223 & 0.0003 & 0.00003 & $\mathrm{D}$ & $\mathrm{z}$ & REST \\
\hline & & Radium-224 & 0.010 & 0.0003 & $\mathrm{D}$ & $\mathrm{z}$ & REST \\
\hline & & Radium-226 & 0.012 & 0.0005 & $\mathrm{D}$ & $\mathrm{z}$ & REST \\
\hline & & Radium-228 & 0.010 & 0.0006 & $\mathrm{D}$ & $\mathrm{z}$ & REST \\
\hline
\end{tabular}


Table 6. Dissolved gases in groundwater from wells sampled in the Marsh Creek valley, Tioga County, Pennsylvania, November 2-4, 2010.

[USGS, U.S. Geological Survey; Gas analyses were performed at Isotech Laboratories, Inc. ND, not detected; NA, not analyzed; mg/L, milligrams per liter; <, less than]

\begin{tabular}{|c|c|c|c|c|c|c|c|}
\hline Constituent & $\begin{array}{l}\text { USGS well name: } \\
\text { Local well name: } \\
\text { Date sampled: } \\
\text { Time sampled: }\end{array}$ & $\begin{array}{c}\text { TI } 269 \\
\text { Well } 1 \\
\text { 11/2/2010 } \\
13: 00\end{array}$ & $\begin{array}{c}\text { TI } 270 \\
\text { Well } 2 \\
11 / 3 / 2010 \\
12: 00\end{array}$ & $\begin{array}{c}\text { TI } 271 \\
\text { Well } 3 \\
\text { 11/4/2010 } \\
\text { 9:00 }\end{array}$ & $\begin{array}{c}\text { TI } 536 \\
\text { private } \\
\text { 11/4/2010 } \\
11: 45\end{array}$ & $\begin{array}{c}\text { TI } 729 \\
\text { private } \\
\text { 11/3/2010 } \\
\text { 9:00 }\end{array}$ & $\begin{array}{c}\text { TI } 730 \\
\text { Well } 5 \\
11 / 2 / 2010 \\
15: 00\end{array}$ \\
\hline \multicolumn{8}{|c|}{$\begin{array}{l}\text { Concentration in headspace, in volume percent } \\
\text { (methane is also expressed as } \mathrm{mg} / \mathrm{L}_{\text {dissolved in water, where noted) }}\end{array}$} \\
\hline Carbon dioxide & & 1.47 & 1.32 & 0.19 & 1.56 & 1.83 & 1.15 \\
\hline Nitrogen & & 77.35 & 75.37 & 76.7 & 75.38 & 77.65 & 75.55 \\
\hline Carbon monoxide & & 0.018 & 0.033 & 0.027 & 0.022 & 0.039 & 0.022 \\
\hline Methane & & ND & ND & 0.015 & 0.002 & 0.341 & ND \\
\hline Propane & & ND & ND & ND & ND & ND & ND \\
\hline Propene & & NA & ND & ND & NA & NA & NA \\
\hline Iso-butane & & ND & ND & ND & ND & ND & ND \\
\hline Normal butane & & ND & ND & ND & ND & ND & ND \\
\hline Iso-pentane & & ND & ND & ND & ND & ND & ND \\
\hline Normal pentane & & ND & ND & ND & ND & ND & ND \\
\hline Hexanes plus & & ND & ND & ND & ND & ND & ND \\
\hline
\end{tabular}

${ }^{1}$ Results for gas composition were normalized by Isotech Laboratories, Inc., to 100 percent and reported in mole percent. Mole percent is approximately equal to volume percent and is reported here as volume percent. 
Table 7. Stable isotopic composition of hydrogen, oxygen, and carbon in groundwater from wells sampled in the Marsh Creek valley, Tioga County, Pennsylvania, November 2-4, 2010.

[USGS, U.S. Geological Survey; Isotopic analyses were performed at Isotech Laboratories, Inc. Stable isotope data reported relative to Vienna Standard Mean Ocean Water (VSMOW) and carbon stable isotope data reported relative to Vienna PeeDee Belemnite (VPDB). $\delta$ D $\mathrm{H}_{2} \mathrm{O}$, hydrogen isotopes of water; $\delta^{18} \mathrm{O} \mathrm{H}_{2} \mathrm{O}$, oxygen isotopes of water; $\delta^{13} \mathrm{C}$ DIC, carbon isotopes of dissolved inorganic carbon]

\begin{tabular}{|c|c|c|c|c|c|c|c|}
\hline \multirow{3}{*}{ Constituent } & USGS well name: & TI 269 & Tl 270 & TI 271 & TI 536 & TI 729 & TI 730 \\
\hline & Local well name: & Well 1 & Well 2 & Well 3 & private & private & Well 5 \\
\hline & Date sampled: & $11 / 2 / 2010$ & $11 / 3 / 2010$ & $11 / 4 / 2010$ & $11 / 4 / 2010$ & $11 / 3 / 2010$ & $11 / 2 / 2010$ \\
\hline \multicolumn{8}{|c|}{ Isotopic composition, in per mil } \\
\hline$\delta \mathrm{D} \mathrm{H}_{2} \mathrm{O}$ & & -67.58 & -67.84 & -66.99 & -67.22 & -70.01 & -69.05 \\
\hline$\delta^{13} \mathrm{C}$ DIC & & -16.53 & -14.79 & -13.92 & -16.77 & -15.01 & -13.89 \\
\hline
\end{tabular}

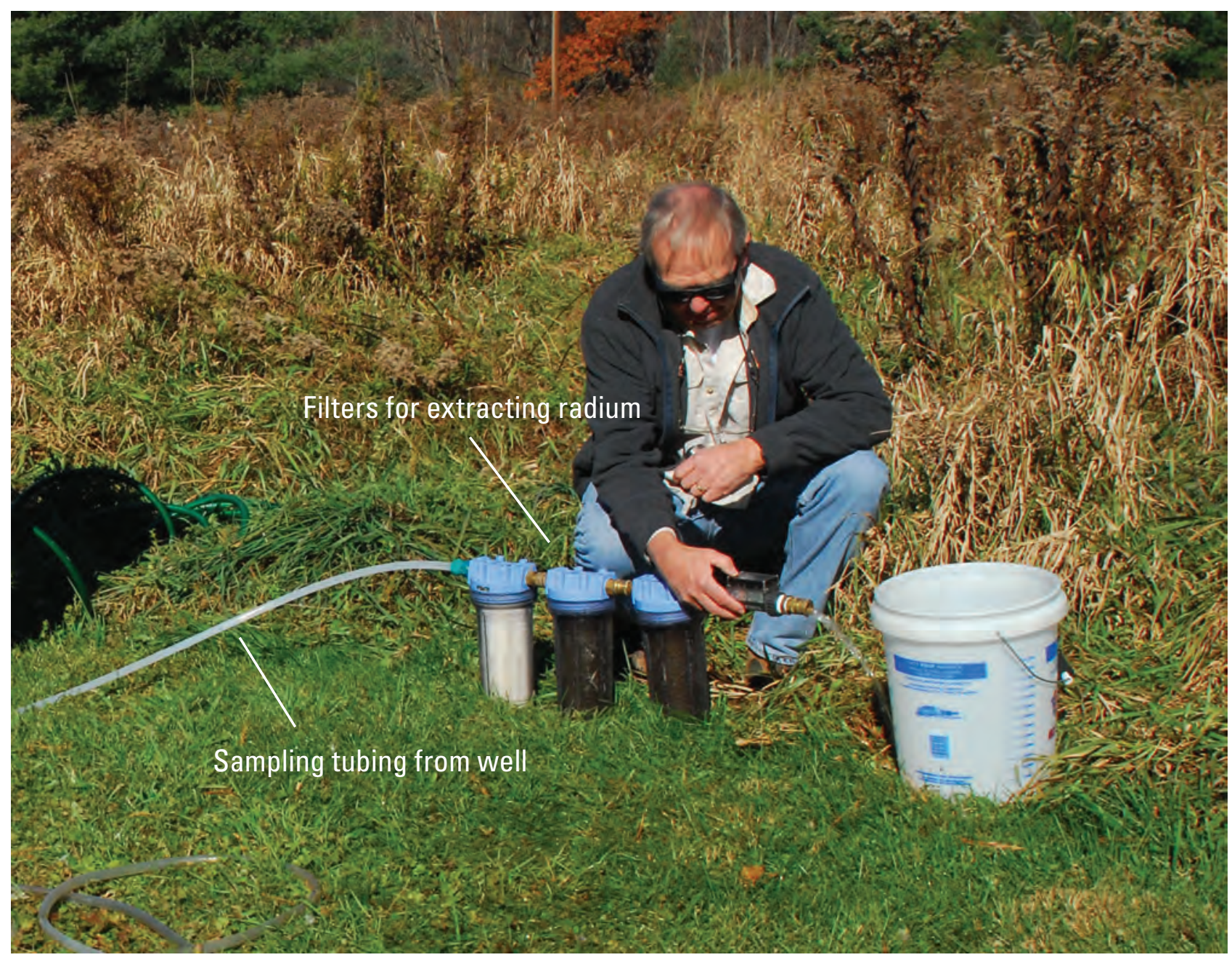

Figure 3. Equipment for sampling groundwater for radium. About 100 gallons of water is flushed through permanganateimpregnated filters that concentrate the radium prior to analysis. 
Table 8. Summary of laboratories, laboratory codes, and methods used to analyze water samples collected in the Marsh Creek valley, Tioga County, Pennsylvania, November 2-4, 2010.

[NWQL, U.S. Geological Survey National Water Quality Laboratory; TDS, total dissolved solids; GC, Gas chromatography; MS, mass spectrometry; ${ }^{\circ} \mathrm{C}$, degrees Celsius; REST, U.S. Geological Survey Radiochemistry Laboratory in Reston, Virginia; IST, Isotech Laboratories, Inc.; ESL, Eberline Services Laboratory]

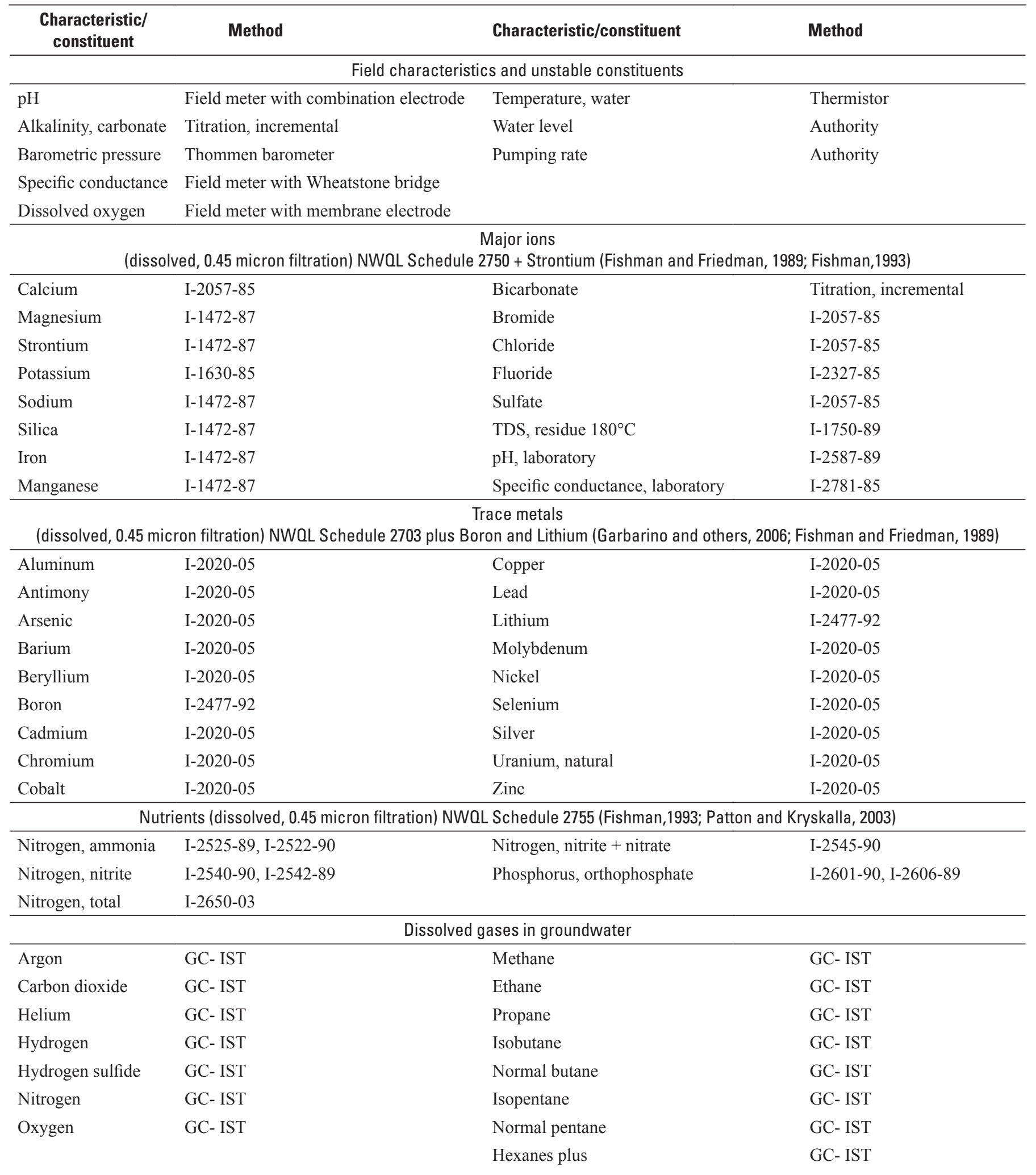


Table 8. Summary of laboratories, laboratory codes, and methods used to analyze water samples collected in the Marsh Creek valley, Tioga County, Pennsylvania, November 2-4, 2010.

[NWQL, U.S. Geological Survey National Water Quality Laboratory; TDS, total dissolved solids; GC, Gas chromatography; MS, mass spectrometry; ${ }^{\circ} \mathrm{C}$, degrees Celsius; REST, U.S. Geological Survey Radiochemistry Laboratory in Reston, Virginia; IST, Isotech Laboratories, Inc.; ESL, Eberline Services Laboratory]

\begin{tabular}{llcc}
\hline $\begin{array}{c}\text { Characteristic/ } \\
\text { constituent }\end{array}$ & \multicolumn{1}{c}{ Method } & Characteristic/constituent & Method \\
\hline & & Isotope ratios (if gases are present) & \\
\hline 2H/1H Methane & MS- IST & 13C/12C Methane & MS- IST \\
& & 13C/12C Carbon dioxide & MS- IST \\
\hline & & Radiochemicals & \\
\hline Gross Alpha Count & Th-230 curve, ESL & Radon-222 & Liquid Scintillation, NWQL-LC1369 \\
Gross Beta Count & Cs-137 curve, ESL & Uranium-234 & Alpha Spectrometry, REST \\
Radium-223 & Alpha Spectrometry, REST & Uranium-238 & Alpha Spectrometry, REST \\
Radium-224 & Alpha Spectrometry, REST & & \\
Radium-226 & Alpha Spectrometry, REST & & \\
Radium-228 & Beta Counting, REST & \\
\hline
\end{tabular}

accreditations, laboratory audits, and proficiency testing are available online at http://nwql.usgs.gov/quality.shtml. Eberline Services, Inc., laboratory is certified by the U.S. Environmental Protection Agency (USEPA). The USGS radiochemical laboratory in Reston, Virginia, is a research facility used in this study to analyze radium and uranium isotopes because very small concentrations of these constituents were anticipated - concentrations well below those commercial laboratories typically report. Isotech Laboratories, Inc., is widely used for analysis of hydrocarbon gases and isotopes and has been chosen by USEPA to analyze samples for their study to evaluate the effect of hydraulic fracturing on drinking-water resources.

\section{Quality Assurance}

Chain-of-custody procedures were used with each laboratory to track samples and ensure sample integrity during shipment, except for the radium and uranium isotopic samples analyzed by the USGS laboratory in Reston, Virginia. The radiochemistry laboratory is a research facility that does not have a chain-of-custody protocol; however, the radiochemical samples were collected, transported, and analyzed by the laboratory director.

One replicate sample was collected from well 2 (TI 270) to show the reproducibility of results (table 9). Results were reproduced within acceptable limits. Results differing by greater than 10 percent were partly the result of comparing the rounded values of very small concentrations. To check the laboratory results for major cations and anions, charge balances were computed and found to range from 0.42 to 5.6 percent, which is acceptable for low ionic-strength waters (Hem, 1985, p. 164).
NWQL performance on the fall 2010 inter-laboratory comparison study is shown in table 10. This study indicates how well the laboratory detected known concentrations of various constituents in double-blind standard-reference samples. NWQL performance was acceptable, except for analyses for vanadium, copper, and nickel; thus, reported concentrations for these constituents should be viewed as less reliable than those for the other analytes.

\section{Simulation of Groundwater Sources to Wells}

The areas contributing recharge and sources of water were estimated for the three high-capacity wells at NARL by use of a two-dimensional, steady-state groundwater model originally developed by Williams and Morrissey (1996) with the use of MODFLOW-88 (McDonald and Harbaugh, 1988). They calibrated the model by comparing simulated streamflow losses in Asaph Run and Straight Run to the average losses determined from streamflow measurements made during various flow conditions during 1985, and by comparing simulated groundwater levels to average levels for 18 wells measured weekly from November 1984 to January 1985 , during a period that was considered by Williams and Morrisey (1996) to approximate long-term average conditions. The hydrologic characteristics were not changed for this study, except for the location and withdrawal rates of the NARL wells. The model was updated to newer software packages as described below.

\section{Updates to the Model}

The model datasets of Williams and Morrissey (1996) were imported into a GIS-based graphical user interface 
Table 9. Analytical results for the environmental sample and the sequential replicate of groundwater from well 2 (TI 270) in the Marsh Creek valley, Tioga County, Pennsylvania, November 3, 2010.

$\left[{ }^{\circ} \mathrm{C}\right.$, degrees Celsius; <, less than; as $\mathrm{N}$, as nitrogen; as $\mathrm{P}$, as phosphorus; $\mathrm{ND}$, reported as not detected by laboratory; N/A, could not compute percent difference; $\mathrm{mg} / \mathrm{L}$, milligrams per liter; $72-\mathrm{h}$, sample analyzed for concentration at approximately 72 hours after sample collection as referenced to a detector calibrated using ${ }^{230} \mathrm{Th}$ for gross alpha and ${ }^{137} \mathrm{Cs}$ for gross beta; $30-\mathrm{d}$, sample used for the 72-hour analysis is counted a second time approximately 30 days after the initial count as referenced to a detector calibrated using ${ }^{230} \mathrm{Th}$ for gross alpha and ${ }^{137} \mathrm{Cs}$ for gross beta; Isotopic analyses were performed at Isotech Laboratories, Inc. Stable isotope data for hydrogen and oxygen are reported relative to Vienna Standard Mean Ocean Water (VSMOW), and stable isotope data for carbon is reported relative to Vienna PeeDee Belemnite (VPDB). dD $\mathrm{H}_{2} \mathrm{O},{ }^{2} \mathrm{H} /{ }^{1} \mathrm{H}$ ratio in water; $\mathrm{d}^{18} \mathrm{O} \mathrm{H}_{2} \mathrm{O},{ }^{18} \mathrm{O} /{ }^{16} \mathrm{O}$ ratio in water; $\mathrm{d}^{13} \mathrm{C}$ DIC, ${ }^{13} \mathrm{C} /{ }^{12} \mathrm{C}$ ratio in dissolved inorganic carbon]

\begin{tabular}{|c|c|c|c|}
\hline Constituent & $\begin{array}{c}\text { TI } 270 \\
\text { (environmental } \\
\text { sample } \\
\text { collected at } \\
\text { 12:00 p.m.) } \\
\end{array}$ & $\begin{array}{c}\text { TI } 270 \\
\text { (replicate } \\
\text { sample } \\
\text { collected at } \\
\text { 12:01 p.m.) }\end{array}$ & $\begin{array}{l}\text { Percent } \\
\text { difference }\end{array}$ \\
\hline \multicolumn{4}{|c|}{ Major lons (milligrams per liter) } \\
\hline Calcium & 10.9 & 10.9 & 0.00 \\
\hline Magnesium & 1.81 & 1.81 & 0.00 \\
\hline Sodium & 1.44 & 1.44 & 0.00 \\
\hline Potassium & 0.78 & 0.80 & -2.56 \\
\hline Chloride & 1.57 & 1.58 & -0.64 \\
\hline Sulfate & 8.33 & 8.32 & 0.12 \\
\hline Fluoride & 0.04 & $<0.04$ & N/A \\
\hline Silica $\left(\right.$ as $\left.\mathrm{SiO}_{2}\right)$ & 4.96 & 5.01 & -1.01 \\
\hline Hardness (as $\left.\mathrm{CaCO}_{3}\right)$ & 34.9 & 34.8 & 0.29 \\
\hline Dissolved solids, sum of constituents & 48 & 48 & 0.00 \\
\hline Dissolved solids dried at $180^{\circ} \mathrm{C}$ & 43 & 49 & -13.95 \\
\hline \multicolumn{4}{|c|}{ Nutrients (milligrams per liter) } \\
\hline Ammonia (as N) & $<0.010$ & $<0.010$ & 0.00 \\
\hline Nitrite (as N) & $<0.001$ & $<0.001$ & 0.00 \\
\hline Nitrate, water (as N) & 0.18 & 0.17 & 5.56 \\
\hline Nitrate plus nitrite (as N) & 0.18 & 0.17 & 5.56 \\
\hline $\begin{array}{l}\text { Total nitrogen (nitrate }+ \text { nitrite }+ \text { ammonia }+ \\
\text { organic-N), as } \mathrm{N}\end{array}$ & 0.18 & 0.17 & 5.56 \\
\hline Orthophosphate (as P) & 0.009 & 0.009 & 0.00 \\
\hline \multicolumn{4}{|c|}{ Trace metals (micrograms per liter) } \\
\hline Arsenic & 0.06 & 0.05 & 16.67 \\
\hline Barium & 12 & 12 & 0.00 \\
\hline Beryllium & $<0.01$ & $<0.01$ & 0.00 \\
\hline Boron & 6 & 6 & 0.00 \\
\hline Bromide & 20 & 10 & 50.00 \\
\hline Cadmium & $<0.02$ & $<0.02$ & 0.00 \\
\hline Chromium & $<0.06$ & $<0.06$ & 0.00 \\
\hline Cobalt & $<0.02$ & $<0.02$ & 0.00 \\
\hline Copper & 41.9 & 44.1 & -5.25 \\
\hline Iron & 5 & 5 & 0.00 \\
\hline Lead & 0.12 & 0.12 & 0.00 \\
\hline
\end{tabular}


Table 9. Analytical results for the environmental sample and the sequential replicate of groundwater from well 2 (TI 270) in the Marsh Creek valley, Tioga County, Pennsylvania, November 3, 2010.

$\left[{ }^{\circ} \mathrm{C}\right.$, degrees Celsius; <, less than; as N, as nitrogen; as P, as phosphorus; ND, reported as not detected by laboratory; N/A, could not compute percent difference; $\mathrm{mg} / \mathrm{L}$, milligrams per liter; 72-h, sample analyzed for concentration at approximately 72 hours after sample collection as referenced to a detector calibrated using ${ }^{230} \mathrm{Th}$ for gross alpha and ${ }^{137} \mathrm{Cs}$ for gross beta; 30 -d, sample used for the 72-hour analysis is counted a second time approximately 30 days after the initial count as referenced to a detector calibrated using ${ }^{230} \mathrm{Th}$ for gross alpha and ${ }^{137} \mathrm{Cs}$ for gross beta; Isotopic analyses were performed at Isotech Laboratories, Inc. Stable isotope data for hydrogen and oxygen are reported relative to Vienna Standard Mean Ocean Water (VSMOW), and stable isotope data for carbon is reported relative to Vienna PeeDee Belemnite (VPDB). dD $\mathrm{H}_{2} \mathrm{O},{ }^{2} \mathrm{H} /{ }^{1} \mathrm{H}$ ratio in water; $\mathrm{d}^{18} \mathrm{O} \mathrm{H}_{2} \mathrm{O},{ }^{18} \mathrm{O} /{ }^{16} \mathrm{O}$ ratio in water; $\mathrm{d}^{13} \mathrm{C}$ DIC, ${ }^{13} \mathrm{C} /{ }^{12} \mathrm{C}$ ratio in dissolved inorganic carbon]

\begin{tabular}{|c|c|c|c|}
\hline Constituent & $\begin{array}{c}\text { TI } 270 \\
\text { (environmental } \\
\text { sample } \\
\text { collected at } \\
\text { 12:00 p.m.) }\end{array}$ & $\begin{array}{c}\text { TI } 270 \\
\text { (replicate } \\
\text { sample } \\
\text { collected at } \\
\text { 12:01 p.m.) }\end{array}$ & $\begin{array}{c}\text { Percent } \\
\text { difference }\end{array}$ \\
\hline \multicolumn{4}{|c|}{ Trace metals (micrograms per liter) } \\
\hline Manganese & 0.4 & 0.4 & 0.00 \\
\hline Molybdenum & $<.03$ & $<.03$ & 0.00 \\
\hline Nickel & $<0.09$ & $<0.09$ & 0.00 \\
\hline Silver & $<0.005$ & $<0.005$ & 0.00 \\
\hline Strontium & 36.5 & 36.9 & -1.10 \\
\hline Zinc & $<1.4$ & $<1.4$ & 0.00 \\
\hline Antimony & $<0.03$ & $<0.03$ & 0.00 \\
\hline Aluminum & $<1.7$ & $<1.7$ & 0.00 \\
\hline Lithium & 0.9 & 0.9 & 0.00 \\
\hline Selenium & 0.05 & 0.04 & 20.00 \\
\hline Uranium (natural) & 0.01 & 0.01 & 0.00 \\
\hline \multicolumn{4}{|c|}{ Radiochemicals (picoCuries per liter) } \\
\hline Gross alpha (72-h) & 0.3 & $<0.3$ & N/A \\
\hline Gross alpha (30-d) & $<0.1$ & ND & N/A \\
\hline Gross beta $(72-\mathrm{h})$ & 1.1 & ND & N/A \\
\hline Gross beta $(30-d)$ & $<0.7$ & $<0.3$ & N/A \\
\hline Radon-222 & 1260 & 1240 & 1.59 \\
\hline Radium-223 & 0.0006 & 0.0006 & 0.87 \\
\hline Radium-224 & 0.007 & 0.007 & -5.82 \\
\hline Radium-226 & 0.007 & 0.007 & 2.78 \\
\hline Radium-228 & 0.008 & 0.008 & 2.85 \\
\hline \multicolumn{4}{|c|}{ Dissolved gases (volume percent in headspace) } \\
\hline Argon & 1.37 & 1.37 & 0.00 \\
\hline Oxygen & 21.91 & 21.92 & -0.05 \\
\hline Carbon Dioxide & 1.32 & 1.36 & -3.03 \\
\hline Nitrogen & 75.37 & 75.32 & 0.07 \\
\hline Carbon Monoxide & 0.033 & 0.026 & 21.21 \\
\hline Methane & ND & ND & N/A \\
\hline Methane, dissolved (mg/L) & $<0.0003$ & $<0.0003$ & N/A \\
\hline Ethane & ND & ND & N/A \\
\hline Ethylene & ND & ND & N/A \\
\hline Propane & ND & ND & N/A \\
\hline
\end{tabular}


Table 9. Analytical results for the environmental sample and the sequential replicate of groundwater from well 2 (TI 270) in the Marsh Creek valley, Tioga County, Pennsylvania, November 3, 2010.

$\left[{ }^{\circ} \mathrm{C}\right.$, degrees Celsius; <, less than; as N, as nitrogen; as P, as phosphorus; ND, reported as not detected by laboratory; N/A, could not compute percent difference; $\mathrm{mg} / \mathrm{L}$, milligrams per liter; 72-h, sample analyzed for concentration at approximately 72 hours after sample collection as referenced to a detector calibrated using ${ }^{230} \mathrm{Th}$ for gross alpha and ${ }^{137} \mathrm{Cs}$ for gross beta; 30 -d, sample used for the 72 -hour analysis is counted a second time approximately 30 days after the initial count as referenced to a detector calibrated using ${ }^{230} \mathrm{Th}$ for gross alpha and ${ }^{137} \mathrm{Cs}$ for gross beta; Isotopic analyses were performed at Isotech Laboratories, Inc. Stable isotope data for hydrogen and oxygen are reported relative to Vienna Standard Mean Ocean Water (VSMOW), and stable isotope data for carbon is reported relative to Vienna PeeDee Belemnite (VPDB). dD $\mathrm{H}_{2} \mathrm{O},{ }^{2} \mathrm{H} /{ }^{1} \mathrm{H}$ ratio in water; $\mathrm{d}^{18} \mathrm{O} \mathrm{H}_{2} \mathrm{O},{ }^{18} \mathrm{O} /{ }^{16} \mathrm{O}$ ratio in water; $\mathrm{d}^{13} \mathrm{C}$ DIC, ${ }^{13} \mathrm{C} /{ }^{12} \mathrm{C}$ ratio in dissolved inorganic carbon]

\begin{tabular}{lccc}
\hline Constituent & $\begin{array}{c}\text { TI 270 } \\
\text { (environmental } \\
\text { sample } \\
\text { collected at } \\
\mathbf{1 2 : 0 0 ~ p . m . )}\end{array}$ & $\begin{array}{c}\text { TI 270 } \\
\text { (replicate } \\
\text { sample } \\
\text { collected at } \\
\mathbf{1 2 : 0 1 ~ p . m . )}\end{array}$ & $\begin{array}{c}\text { Percent } \\
\text { difference }\end{array}$ \\
\hline Dissolved gases (volume percent in headspace) & \\
\hline Iso-butane & $\mathrm{ND}$ & $\mathrm{ND}$ & $\mathrm{N} / \mathrm{A}$ \\
Normal butane & $\mathrm{ND}$ & $\mathrm{ND}$ & $\mathrm{N} / \mathrm{A}$ \\
Iso-pentane & $\mathrm{ND}$ & $\mathrm{ND}$ & $\mathrm{N} / \mathrm{A}$ \\
Normal pentane & $\mathrm{ND}$ & $\mathrm{ND}$ & $\mathrm{N} / \mathrm{A}$ \\
Hexanes plus & $\mathrm{ND}$ & $\mathrm{ND}$ & $\mathrm{N} / \mathrm{A}$ \\
& $\mathrm{ND}$ & $\mathrm{ND}$ & $\mathrm{N} / \mathrm{A}$ \\
\hline$\delta \mathrm{D} \mathrm{H} \mathrm{H}_{2} \mathrm{O}$ & Isotopic composition (per mil) & & \\
$\delta^{18} \mathrm{O} \mathrm{H}_{2} \mathrm{O}$ & -67.84 & -67.74 & 0.14 \\
$\delta^{13} \mathrm{C} \mathrm{DIC}$ & -9.95 & -10.02 & -0.70 \\
\hline
\end{tabular}


Table 10. Performance of the U.S. Geological Survey National Water Quality Laboratory in analyzing standard-reference samples in the fall 2010 inter-laboratory comparison study.

[NWQL, National Water Quality Laboratory; mg/L, milligrams per liter; $\mu \mathrm{g} / \mathrm{L}$, micrograms per liter; <, less than; as N, as nitrogen; as $\mathrm{P}$, as phosphorus; $\mu \mathrm{S} / \mathrm{cm}$, microsiemens per centimeter at 25 degrees Celsius; --, could not compute percent difference; bold font indicates NWQL result is outside of acceptable deviation from most probable value]

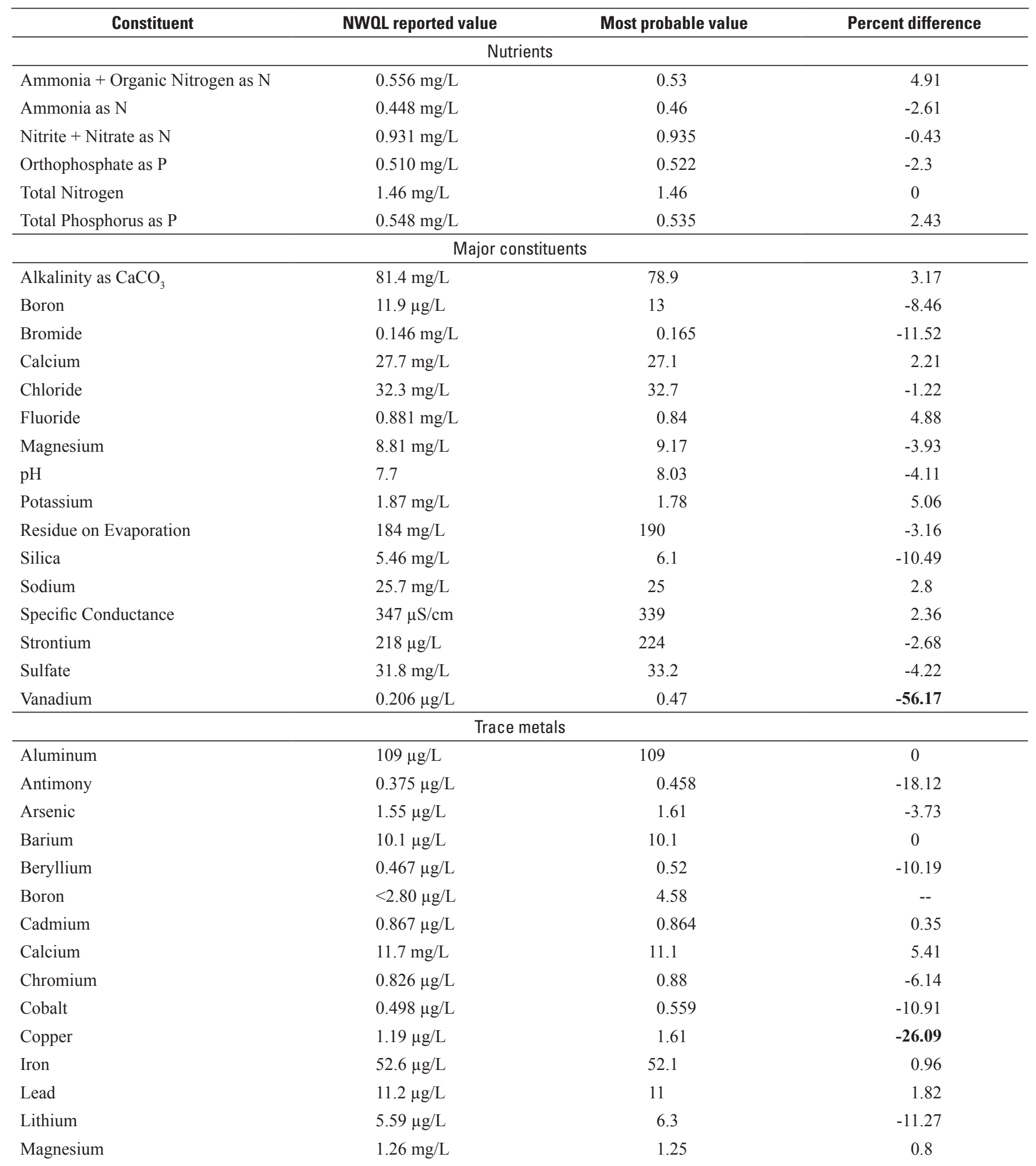


Table 10. Performance of the U.S. Geological Survey National Water Quality Laboratory in analyzing standard-reference samples in the fall 2010 inter-laboratory comparison study.

[NWQL, National Water Quality Laboratory; mg/L, milligrams per liter; $\mu \mathrm{g} / \mathrm{L}$, micrograms per liter; <, less than; as N, as nitrogen; as P, as phosphorus; $\mu \mathrm{S} / \mathrm{cm}$, microsiemens per centimeter at 25 degrees Celsius; --, could not compute percent difference; bold font indicates NWQL result is outside of acceptable deviation from most probable value]

\begin{tabular}{lccc}
\hline \multicolumn{1}{c}{ Constituent } & NWOL reported value & Most probable value & Percent difference \\
\hline Manganese & \multicolumn{2}{c}{ Trace metals } & \\
Molybdenum & $122 \mu \mathrm{g} / \mathrm{L}$ & 127 & -3.94 \\
Nickel & $4.32 \mu \mathrm{g} / \mathrm{L}$ & 4.11 & 5.11 \\
Potassium & $0.238 \mu \mathrm{g} / \mathrm{L}$ & 0.402 & $-\mathbf{4 0 . 8}$ \\
Selenium & $1.30 \mathrm{mg} / \mathrm{L}$ & 1.22 & 6.56 \\
Silica & $0.345 \mu \mathrm{g} / \mathrm{L}$ & 0.397 & -13.1 \\
Silver & $4.90 \mathrm{mg} / \mathrm{L}$ & 4.71 & 4.03 \\
Sodium & $2.56 \mu \mathrm{g} / \mathrm{L}$ & 2.76 & -7.25 \\
Strontium & $10.2 \mathrm{mg} / \mathrm{L}$ & 10.8 & -5.56 \\
Thallium & $43.9 \mu \mathrm{g} / \mathrm{L}$ & 45.5 & -3.52 \\
Uranium & $1.91 \mu \mathrm{g} / \mathrm{L}$ & 1.95 & -2.05 \\
Vanadium & $0.681 \mu \mathrm{g} / \mathrm{L}$ & 0.75 & -9.2 \\
Zinc & $1.07 \mu \mathrm{g} / \mathrm{L}$ & 1.1 & -2.73 \\
& $35.0 \mu \mathrm{g} / \mathrm{L}$ & 36 & -2.78 \\
\hline
\end{tabular}

linked to Argus Numerical Environments (ARGUS) software (Winston, 2000) and reformatted for use with MODFLOW-2000 (Harbaugh and others, 2000) and MODPATH (Pollock, 1994), a particle-tracking program commonly used to delineate contributing recharge areas. The two-dimensional finite-difference grid was georeferenced on the basis of figure 6 in Williams and Morrissey (1996).

The modeled area, two-dimensional finite-difference grid, and hydrologic boundary conditions are shown in figure 4 . The model is oriented N. $67^{\circ} \mathrm{E}$., and grid cells are $200 \mathrm{ft}$ by $200 \mathrm{ft}$ squares that define the active model area, which is bounded by bedrock valley walls, except where crossing Straight Run, Asaph Run, and Marsh Creek. The model has one layer, specified as unconfined. The bottom of the layer represents the altitude of the base of the glacial sand and gravel aquifer ranging from 1,035 to $1,180 \mathrm{ft}$ above the National Geodetic Vertical Datum of 1929 (NGVD 29). Hydraulic conductivity ranges from 52 to 311 feet squared per day $\left(\mathrm{ft}^{2} / \mathrm{d}\right)$. General-head boundaries were assigned across the Marsh Creek valley to simulate groundwater inflow and outflow. Recharge from precipitation on the valley floor was simulated as a constant flux by use of the recharge package in MODFLOW-2000. Streams were simulated with the stream package of Prudic (1989) with streambed-conductance values calibrated against measured seepage rates from Straight Run and Asaph Run (Williams and Morrissey, 1996). Unchanneled upland runoff and withdrawals from the NARL wells and a new well were simulated with constant-flux cells by use of the well package.
All the MODFLOW-2000 data sets were regenerated using ARGUS, except the data for the stream package. The stream-package data set produced by ARGUS could not exactly reproduce the original data set without considerable manipulation of the stream-segment data, so the streampackage dataset from Williams and Morrissey (1996) was used directly. This resulted in a MODFLOW-2000 model of the study area that exactly produced the output of Williams and Morrissey (1996) with results that could be plotted by use of a geographic information system. A summary of the model documentation and available calibration is provided in Williams and Morrisey (1996).

\section{Steady-State Simulation Inputs and Parameters}

Using the previously calibrated model inputs and parameters, eight simulations were conducted to delineate the areas contributing recharge and sources of water to wells for steady-state conditions (table 11). Simulated recharge, streamflow, and pumping rates were varied to illustrate the range in extent of the areas contributing recharge to wells. The "average" and "dry" hydrologic conditions were defined by Williams and Morrissey (1996, p. 101). The conditions from November 1984 through January 1985 were chosen by them as an approximation of long-term average conditions, and the conditions during October 1984 were chosen to represent low-flow (or dry) conditions. Recharge rates applied to the model were 18.9 inches per year (in/yr) for average conditions and $2.7 \mathrm{in} / \mathrm{yr}$ for dry conditions. Because streamflow in Asaph Run and Straight Run was measured for 

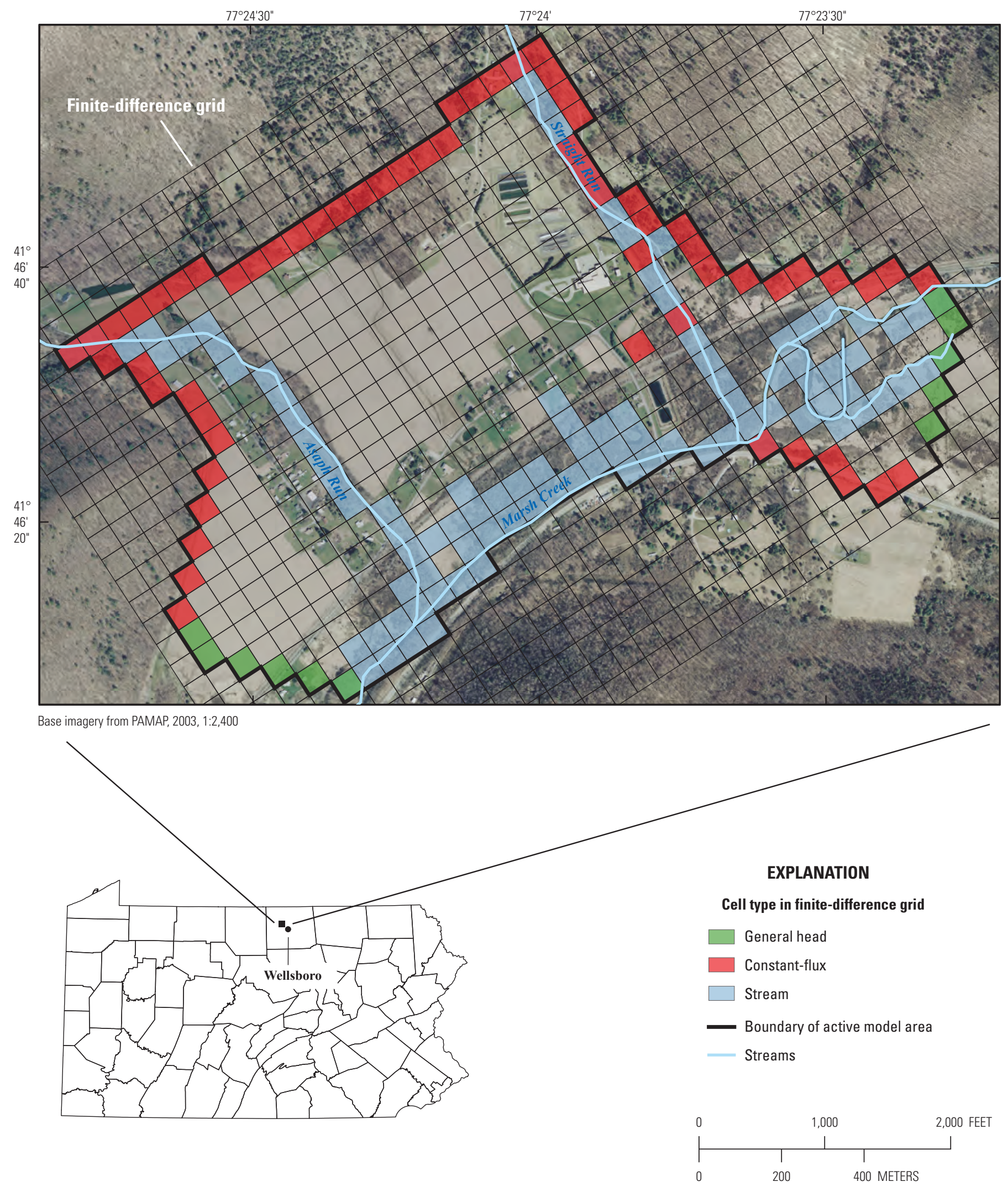

Figure 4. Finite-difference grid and boundary conditions for the groundwater-flow model of Marsh Creek valley, Tioga County, Pennsylvania. 
Table 11. Hydrologic conditions and pumping rates simulated by the steady-state groundwater-flow model of Marsh Creek valley, near Wellsboro, Tioga County, Pennsylvania.

[NARL, Northern Appalachian Research Laboratory]

\begin{tabular}{|c|c|c|c|c|c|}
\hline $\begin{array}{c}\text { Simulation } \\
\text { number }\end{array}$ & $\begin{array}{l}\text { Hydrologic } \\
\text { conditions }\end{array}$ & $\begin{array}{l}\text { Total pumping rate } \\
\text { from three } \\
\text { NARL wells } \\
\text { (gallons per minute) }\end{array}$ & $\begin{array}{c}\text { Proposed } \\
\text { withdrawals } \\
\text { from the new well } \\
\text { (gallons per minute) }\end{array}$ & $\begin{array}{c}\text { Simulated flow } \\
\text { in headwater of } \\
\text { Asaph Run } \\
\text { (gallons per minute) }\end{array}$ & $\begin{array}{l}\text { Simulated flow } \\
\text { in headwater of } \\
\text { Straight Run } \\
\text { (gallons per minute) }\end{array}$ \\
\hline 1 & Average & 1,000 & 0 & 9,694 & 4,663 \\
\hline 2 & Average & 2,000 & 0 & 9,694 & 4,663 \\
\hline 3 & Average & 2,000 & 1,000 & 9,694 & 4,663 \\
\hline 5 & Dry & 1,000 & 0 & 1,616 & 494 \\
\hline 6 & Dry & 1,000 & 1,000 & 1,616 & 494 \\
\hline 7 & Dry & 2,000 & 0 & 1,616 & 494 \\
\hline 8 & Dry & 2,000 & 1,000 & 1,616 & 494 \\
\hline
\end{tabular}

only 18 months during 1984-85, the long-term average and dry conditions cannot be computed for the study area, but insights can be gained by analysis of the streamflow record from Corey Creek near Mainesburg (a small stream in Tioga County about 20 miles east of NARL, not shown on maps) during 1954-2009. Average streamflow in Corey Creek during November 1984 through January 1985 corresponded to the 60th percentile of all monthly mean streamflow values for that creek from 1954 to 2009; the streamflow during October 1984 corresponded to the $22 \mathrm{~d}$ percentile of all monthly mean streamflow values. Thus, the average conditions simulated in the model probably represent wetter than median conditions (60th percentile), and the dry period represents conditions that could be expected 2 to 3 months per year ( $22 \mathrm{~d}$ percentile), not a severe drought. The groundwater budgets for average and dry conditions (without withdrawals from wells) are shown in figure 5. For average conditions, the total simulated groundwater flow through the study area is $4,820 \mathrm{gal} / \mathrm{min}$; for dry conditions, the total simulated groundwater flow through the study area is $2,340 \mathrm{gal} / \mathrm{min}$.

Groundwater withdrawals from the NARL wells 1, 3, and 5 (TI 269, TI 271, and TI 730, respectively) were simulated in the model as steady, equal withdrawal rates from each well. Currently (2011) withdrawals from the three wells total about $1,000 \mathrm{gal} / \mathrm{min}$; thus, simulations were conducted for that rate and for $2,000 \mathrm{gal} / \mathrm{min}$, which is the maximum withdrawal permitted unless the flow of Straight Run is gaged and effects on the wetland along Marsh Creek are monitored (Susquehanna River Basin Commission, 2003). If those actions are taken, NARL is permitted to withdraw an amount equal to the flow of Straight Run but not to exceed $3,000 \mathrm{gal} / \mathrm{min}$. Therefore, pumping 3,000 gal/min was simulated for the average hydrologic condition but not for the dry condition because the simulated flow of Straight Run is only $500 \mathrm{gal} / \mathrm{min}$ entering the modeled area during dry conditions. Therefore, withdrawals greater than 2,000 gal/min would not be allowed. Simulations also were made to evaluate the effects of proposed withdrawals of $1,000 \mathrm{gal} / \mathrm{min}$ from a new well, located about 3,500 ft southwest of the NARL facility.

The sources of water and hydraulic response of the system were estimated from particle tracking by the use of MODPATH. The results are approximate because some of the sinks are characterized as "weak" sinks, which imparts ambiguity as to exactly which particles should be stopped and which should be allowed to pass through the sink. For this study, all particles entering a cell were stopped at that cell if more than half of the flow entering it discharged to a well or stream.

\section{Groundwater Quality in Marsh Creek Valley}

Results of water-quality analyses indicate that samples from the wells in the glacial sand and gravel and bedrock aquifers contained high quality, low-ionic strength water, with less than 80 milligrams per liter $(\mathrm{mg} / \mathrm{L})$ of dissolved solids (table 3). The major-ion chemistry indicates that the samples were all calcium-bicarbonate type waters, except for the sample from TI 729, which contained more sodium than calcium. The groundwater is described as "soft" on the basis of measured hardness (Hem, 1985, p. 159). Nutrient concentrations were far below the maximum contaminant levels (MCLs) established for drinking water in Pennsylvania (Pennsylvania Department of Environmental Protection, 2006).

Concentrations of trace metals and most radiochemicals also were low; some trace metals are listed as non-detects at low levels (table 4). All trace metals and radiochemicals were less than the MCLs. Trace-metal concentrations of aluminum, 


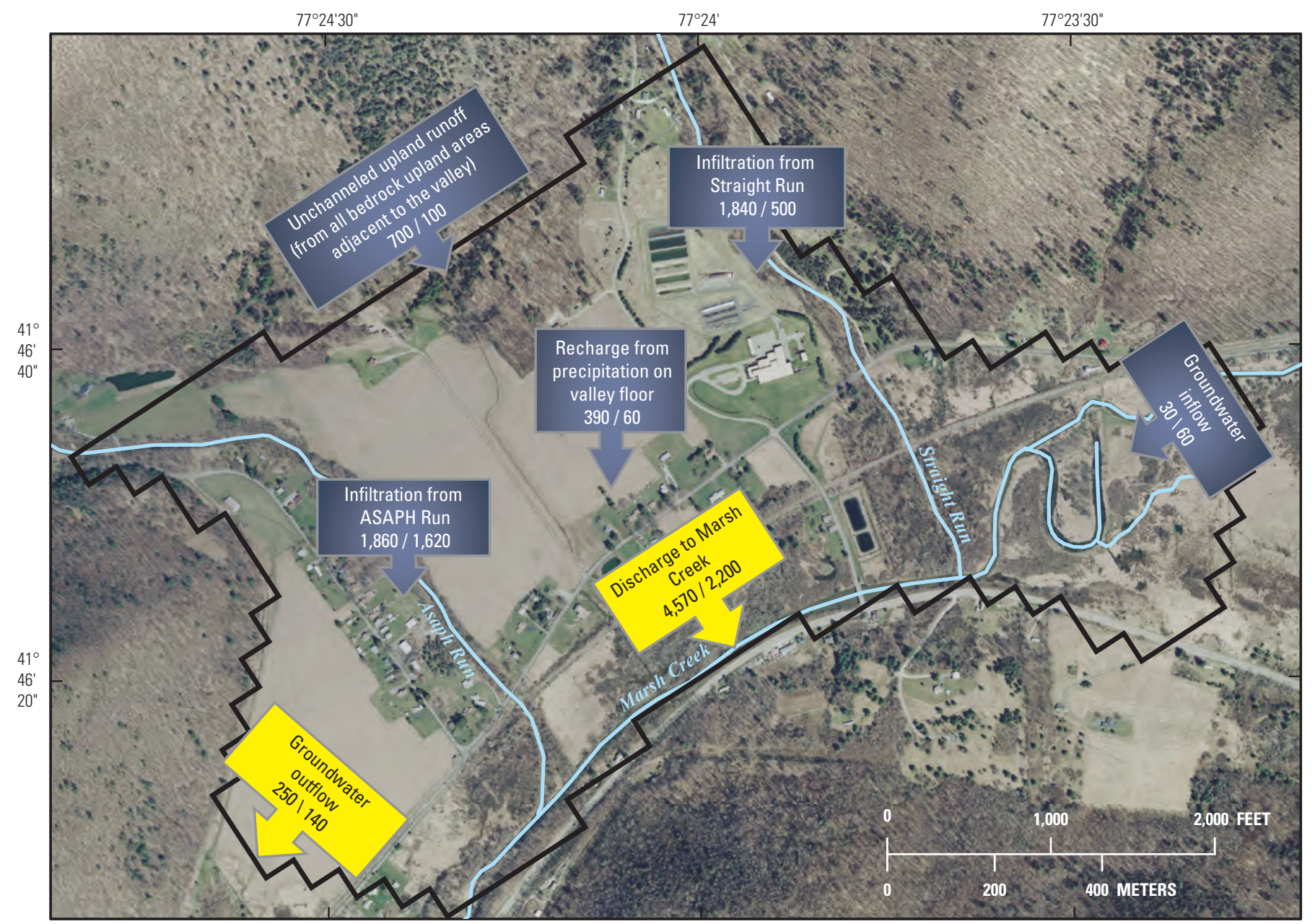

Base imagery from PAMAP, 2003, 1:2,400

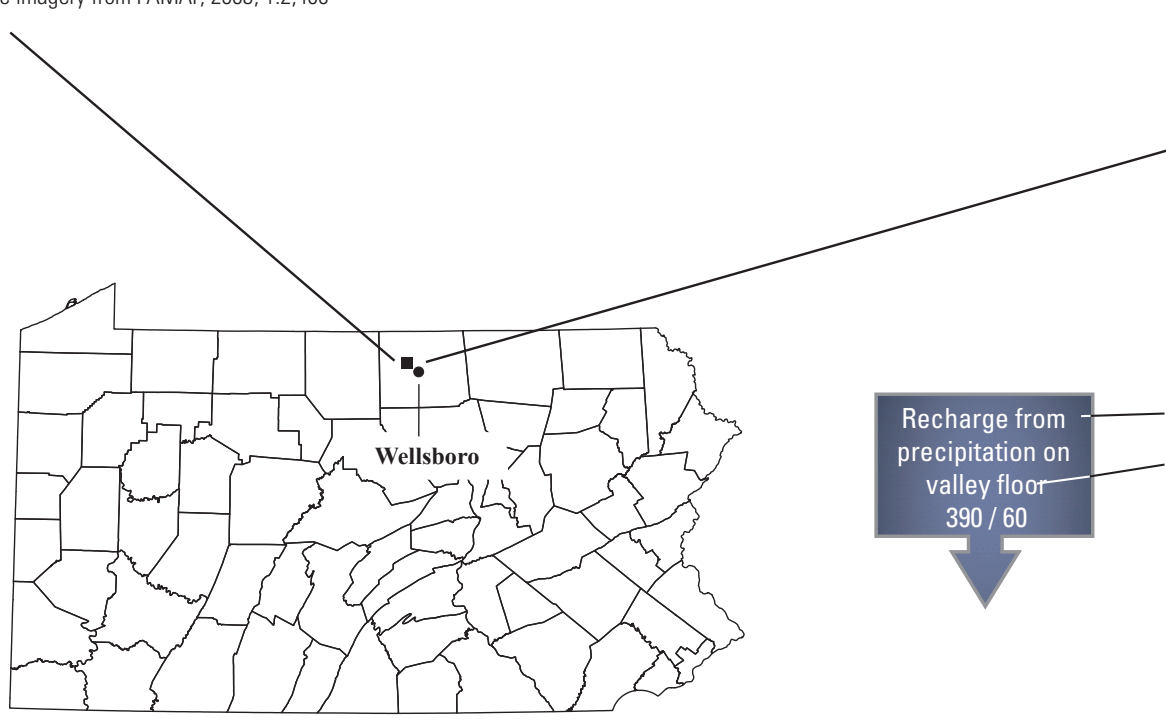

EXPLANATION

Groundwater budget term

Average conditons / Dry conditions-Flow rate, in gallons per minute

Blue represents inflow to groundwater system. Yellow represents ouflow from groundwater system

Figure 5. Steady-state groundwater budgets for average and dry hydrologic conditions without groundwater withdrawals simulated by the groundwater-flow model of Marsh Creek valley, Tioga County, Pennsylvania. 
iron, lithium, nickel, and zinc were greater in water from the domestic-supply wells completed in the bedrock aquifer than from the NARL wells completed in the glacial sand and gravel aquifer. The concentration of copper was greatest in the sample from well 2 (TI 270), possibly because the sample was collected from a tank in the NARL building far from the wellhead and had more contact with copper pipes than did the other samples.

Radon gas was present in the wells at concentrations ranging from 980 to 1,310 picocuries per liter (table 4). Although there currently is not an MCL for radon in drinking water in Pennsylvania, the U.S. Environmental Protection Agency (USEPA) proposed MCL is $300 \mathrm{pCi} / \mathrm{L}$, with an alternate $\mathrm{MCL}$ of $4,000 \mathrm{pCi} / \mathrm{L}$ if an indoor-air mitigation program has been enacted by the State or water purveyor (U.S. Environmental Protection Agency, 1999). Although the radon concentrations found in the wells are elevated compared to the proposed USEPA MCL of $300 \mathrm{pCi} / \mathrm{L}$, they are similar to natural levels found in the glacial sand and gravel deposits and fractured bedrock of the Catskill Formation in other areas of northern Pennsylvania (Senior, 2009, p. 52).

Concentrations of dissolved gases are listed in table 6 . Dissolved gases are reported as volume percent in headspace, which is the reporting convention used by the laboratory. Methane is also reported in table 6 as the concentration dissolved in water, in milligrams per liter.

Concentrations of headspace gases were similar to concentrations found in the atmosphere or soil. Methane was the only hydrocarbon gas detected. It was found in water from NARL well 3 (TI 271) and domestic-supply wells TI 536 and TI 729 at concentrations ranging from 0.0006 to $0.07 \mathrm{mg} / \mathrm{L}$ as dissolved methane in water. These concentrations are low. A methane concentration of $10 \mathrm{mg} / \mathrm{L}$ in groundwater is considered a warning level as to the possibility of accumulation in air that could lead to a subsequent explosion (Eltschlager and others, 2001, p. 40). Osborn and others (2011) reported an average methane concentration of $1.9 \mathrm{mg} / \mathrm{L}$ in five groundwater samples collected from wells in the Catskill Formation. The concentrations of methane from the NARL samples were too low to allow a determination of the isotopic composition of carbon and hydrogen atoms in the methane molecule, which can be useful for determining the origin of the gas.

\section{Sources of Water from Steady- State Simulations}

Eight simulations were made to identify the sources of water contributing to the NARL wells and a new well. The results are presented in the order shown in table 11. Figures in the following sections illustrate the simulation results. Travel time from source area to well discharge point is described. Finally, the assumptions and limitations of the model simulations are discussed.

\section{Average Conditions with 1,000 Gallons per Minute Withdrawals}

Simulations were made for average hydrologic conditions with groundwater withdrawals totaling $1,000 \mathrm{gal} / \mathrm{min}$ from the NARL wells. The areas contributing recharge to each well and the configuration of the water table are illustrated in figure 6A. The wells capture water from a small area that directs stream infiltration from Straight Run to the wells. Infiltration from Straight Run accounts for about 99 percent of the groundwater withdrawn by the wells during average conditions (fig. 6B). The capture of water by the wells affects the water budget of the study area by decreasing the groundwater discharge to Marsh Creek by about $360 \mathrm{gal} / \mathrm{min}$ (36 percent of $1,000 \mathrm{gal} / \mathrm{min}$ ) and increasing seepage from Straight Run by about $640 \mathrm{gal} / \mathrm{min}$ (fig. 6C). The ultimate effect on Marsh Creek downstream from the NARL, however, is negligible because the water use is nearly 100 percent non-consumptive and the water is discharged about $400 \mathrm{ft}$ downstream from the confluence of Straight Run and Marsh Creek.

\section{Average Conditions with 2,000 Gallons per Minute Withdrawals}

Simulations were made for average hydrologic conditions with groundwater withdrawals totaling 2,000 gal $/ \mathrm{min}$ from the NARL wells. The areas contributing recharge to each well and the configuration of the water table are illustrated in figure 7A. Even at 2,000 gal $/ \mathrm{min}$, the wells capture water from only a small area; stream infiltration from Straight Run is directed to the wells. Infiltration from Straight Run accounts for about 98 percent of the groundwater withdrawn by the wells during average hydrologic conditions (fig. 7B). This capture affects the water budget of the study area by decreasing the groundwater discharge to Marsh Creek by about $680 \mathrm{gal} / \mathrm{min}$ (34 percent of 2,000 gal $/ \mathrm{min}$ ) and increasing seepage from Straight Run by about 1,320 gal/min (fig. 7C). The ultimate effect on Marsh Creek, however, is negligible because the groundwater withdrawals are discharged about $400 \mathrm{ft}$ downstream from the confluence of Straight Run and Marsh Creek.

\section{Average Conditions with 2,000 Gallons per Minute Withdrawals Plus 1,000 Gallons per Minute Withdrawal from the New Production Well}

Simulations were made for average hydrologic conditions with groundwater withdrawals totaling 2,000 gal $/ \mathrm{min}$ from the NARL wells and a new withdrawal of $1,000 \mathrm{gal} / \mathrm{min}$ from the production well 3,500 $\mathrm{ft}$ to the southwest. The areas contributing recharge to each well and the configuration of the water table are illustrated in figure $8 \mathrm{~A}$. The area throughout which recharge is captured for the NARL wells is essentially 


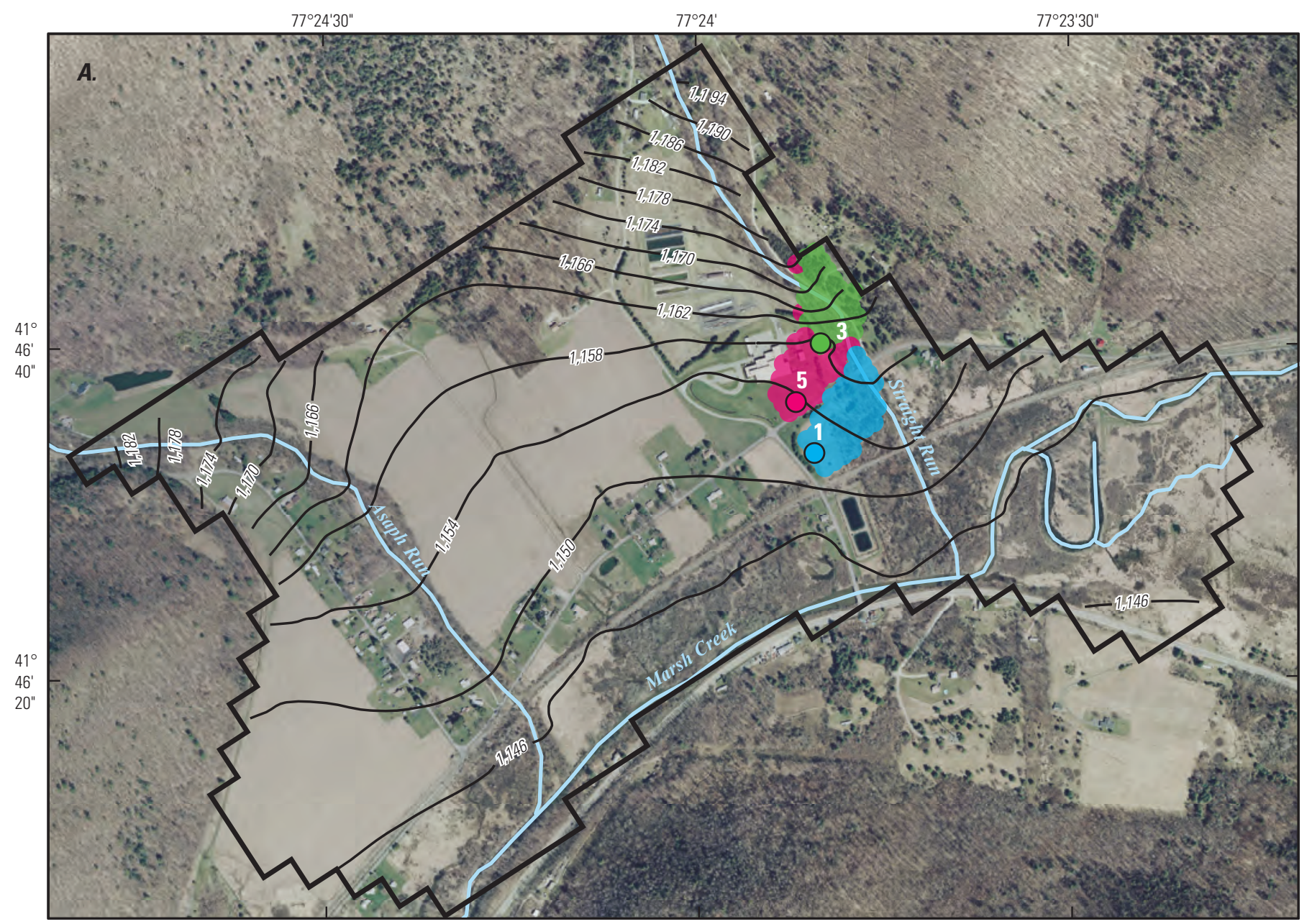

Base imagery from PAMAP, 2003, 1:2,400

\section{EXPLANATION}

$-1,150$ - Water-table contour, in feet

B. Sources of water

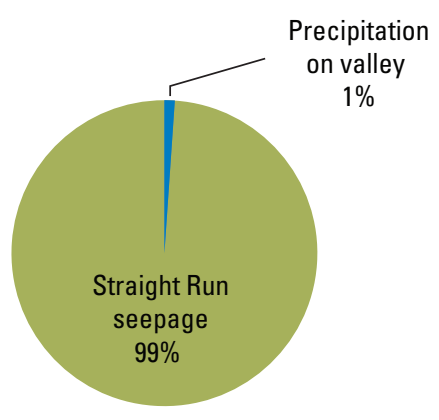

C. Hydraulic response

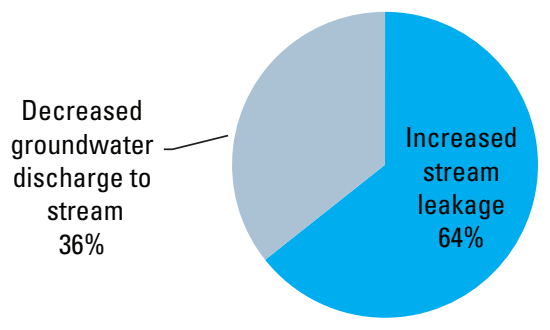
above NGVD 29. Interval 4 feet

Simulated contributing areas and location of well and identifier

Well 3

Well 5

Well 1

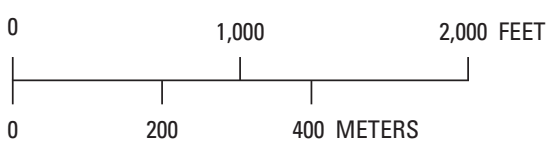

Figure 6. Simulated $A$, areas contributing recharge to wells and water-table contours, $B$, sources of water to wells, and $C$, hydraulic response of the aquifer during average hydrologic conditions with withdrawals of 1,000 gallons per minute from the Northern Appalachian Research Laboratory (NARL) wells, Tioga County, Pennsylvania. 


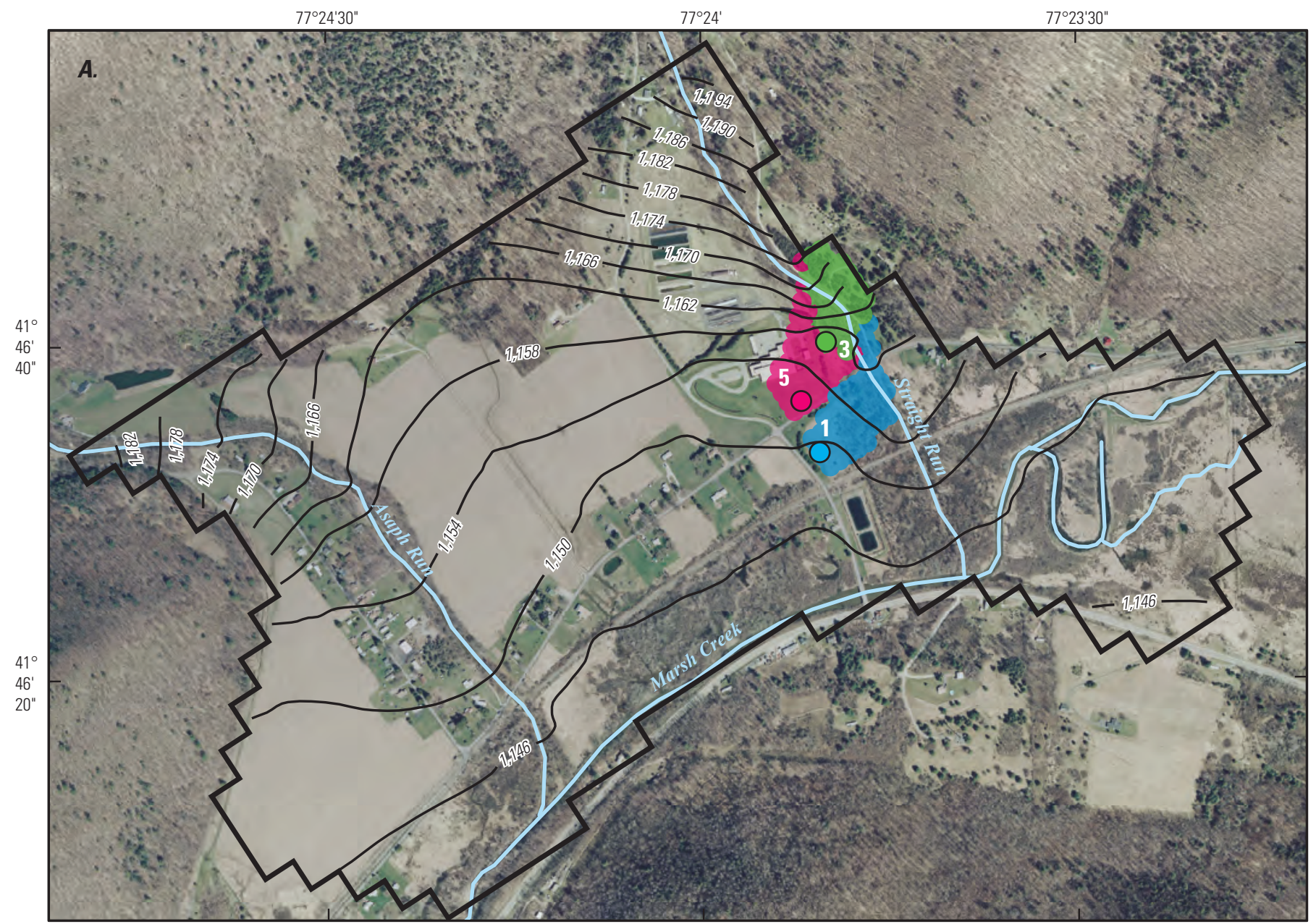

Base imagery from PAMAP, 2003, 1:2,400

B. Sources of water

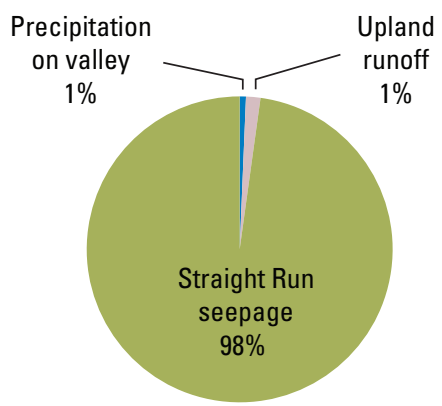

C. Hydraulic response

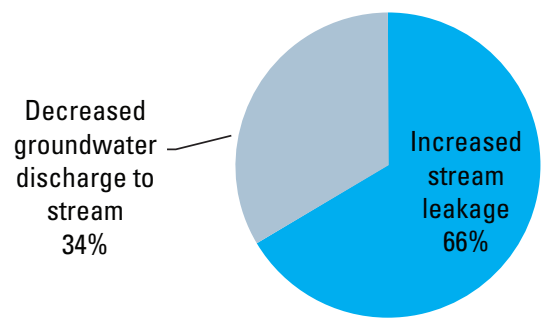

\section{EXPLANATION}

- 1,150 - Water-table contour, in feet above NGVD 29. Interval 4 feet

Simulated contributing areas and location of well and identifier

Well 3

Well 5

Well 1

Figure 7. Simulated $A$, areas contributing recharge to wells and water-table contours, $B$, sources of water to wells, and $C$, hydraulic response of the aquifer during average hydrologic conditions with withdrawals of 2,000 gallons per minute from the Northern Appalachian Research Laboratory (NARL) wells, Tioga County, Pennsylvania. 


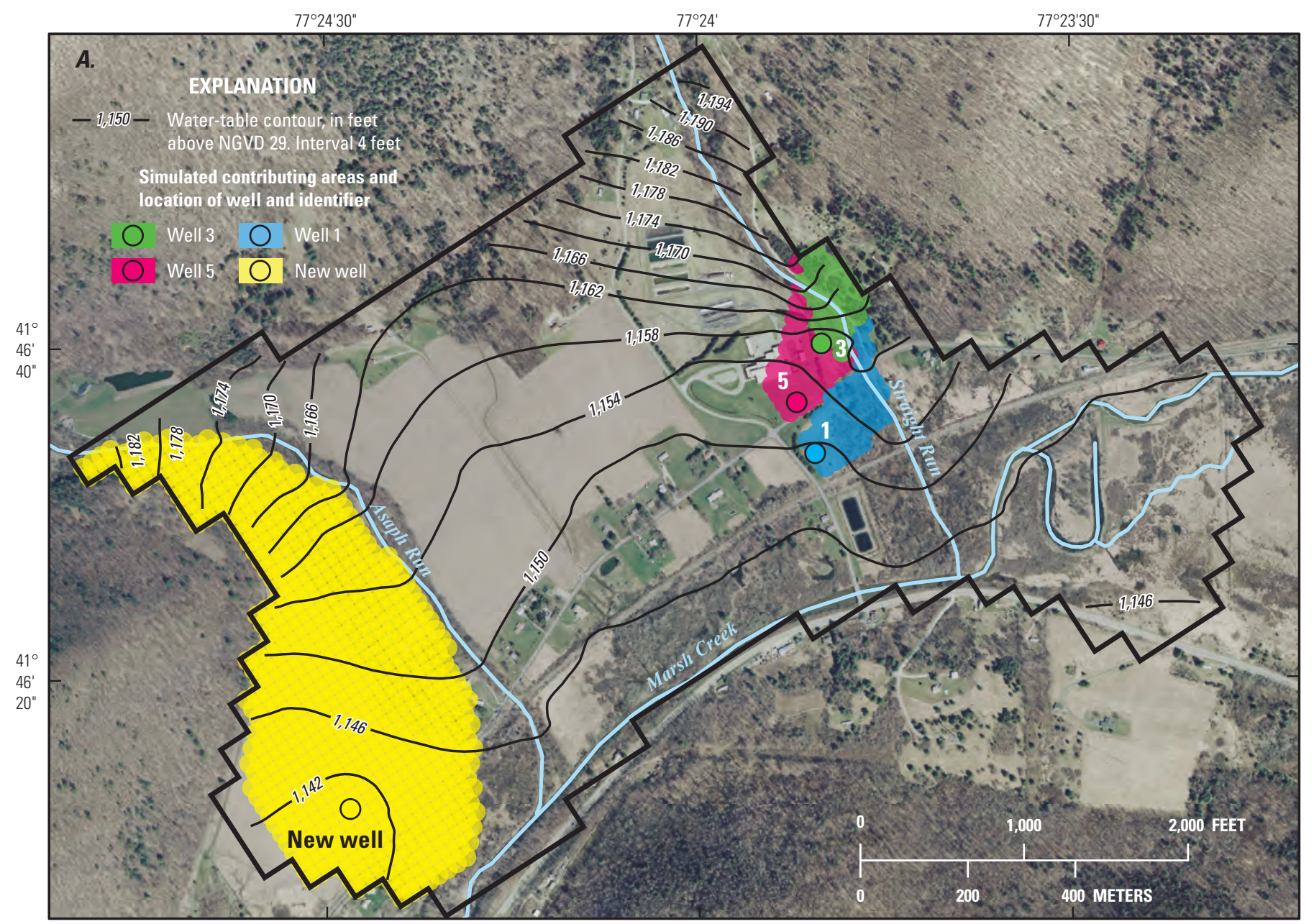

Base imagery from PAMAP, 2003, 1:2,400

\section{B. Sources of water-Northern Appa- lachian Research Laboratory wells}

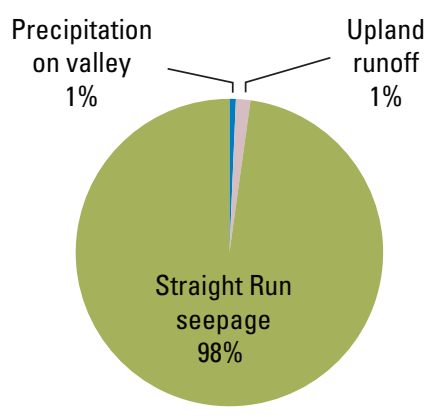

B. Sources of water-New well

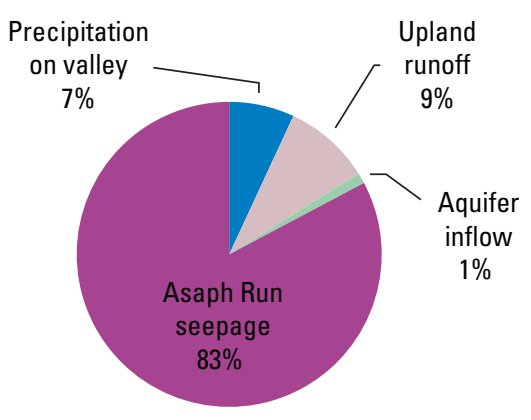

C. Hydraulic response

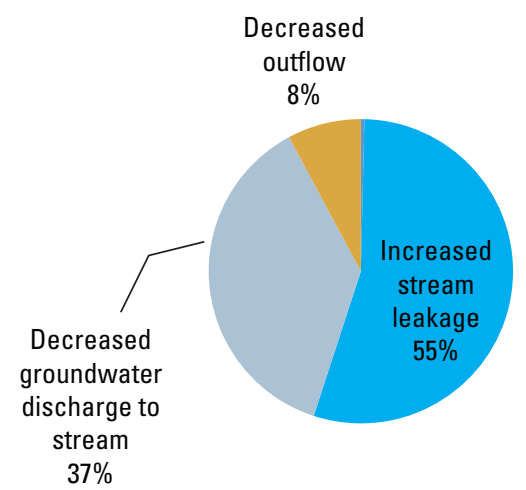

Figure 8. Simulated $A$, areas contributing recharge to wells and water-table contours, $B$, sources of water to wells, and $C$, hydraulic response of the aquifer during average hydrologic conditions with withdrawals of 2,000 gallons per minute from the Northern Appalachian Research Laboratory (NARL) wells and 1,000 gallons per minute from the new well, Tioga County, Pennsylvania. 
unchanged by the addition of the new well (compare figures $7 \mathrm{~A}$ and $8 \mathrm{~A}$ ). The area contributing recharge to the new well extends from Asaph Run to the western boundary of the glacial sand and gravel aquifer. Seepage from Asaph Run is the major source of water to the new well, providing 83 percent of water withdrawals (fig. 8B). The sources of groundwater withdrawn by the NARL wells are the same as if the new well was not operating (see fig. 7B for sources). Capture from these sources affects the water budget of the study area by decreasing the groundwater discharge to Marsh Creek about $1,110 \mathrm{gal} / \mathrm{min}$ (37 percent of 3,000 gal $/ \mathrm{min}$ ) and increasing seepage from Straight Run and Asaph Run by about $1,650 \mathrm{gal} / \mathrm{min}$ (55 percent of $3,000 \mathrm{gal} / \mathrm{min}$; fig. $8 \mathrm{C}$ ). The ultimate effect on Marsh Creek downstream from the NARL, however, is caused only by the consumptive withdrawal from the new well because groundwater withdrawals from the NARL wells are discharged about $400 \mathrm{ft}$ downstream from the confluence of Straight Run and Marsh Creek.

Results of model simulations indicate that pumping $1,000 \mathrm{gal} / \mathrm{min}$ of water from the new well will not cause any substantial additional drawdown in the NARL well field during average hydrologic conditions (fig. 9). The magnitude of the drawdown, however, might be greater than the twodimensional model results indicate because the vertical hydraulic conductivity of the glacial sand and gravel aquifer between the well screen depth and Asaph Run is not simulated by the one-layer model.

\section{Average Conditions with 3,000 Gallons per Minute Withdrawals Plus 1,000 Gallons per Minute Withdrawal from the New Production Well}

Simulations were made for average hydrologic conditions with groundwater withdrawals totaling $3,000 \mathrm{gal} / \mathrm{min}$ from the NARL wells and a new withdrawal of $1,000 \mathrm{gal} / \mathrm{min}$ from the production well 3,500 $\mathrm{ft}$ to the southwest. The areas contributing recharge to each well and the configuration of the water table are illustrated in figure 10A. The increased area in which recharge is captured for the NARL wells is barely discernible when the total withdrawals are increased from $2,000 \mathrm{gal} / \mathrm{min}$ to $3,000 \mathrm{gal} / \mathrm{min}$ (compare figures $8 \mathrm{~A}$ and 10A). Seepage from Asaph Run is the major source of water to the new well, providing 83 percent of the water withdrawn (fig. 10B). The sources of groundwater withdrawn by the NARL wells are the same as those shown in figure 7B. Capture from these sources affects the water budget of the study area by decreasing the groundwater discharge to Marsh Creek by about $1,480 \mathrm{gal} / \mathrm{min}$ ( 37 percent of $4,000 \mathrm{gal} / \mathrm{min}$ ) and increasing seepage from Straight Run and Asaph Run by about 2,280 gal/min (57 percent of 4,000 gal $/ \mathrm{min}$; fig. 10C). The ultimate effect on streamflow in Marsh Creek downstream from the NARL is caused only by the consumptive withdrawal of $1,000 \mathrm{gal} / \mathrm{min}$ from the new well; withdrawals from the
NARL wells are returned as wastewater discharge to Marsh Creek about $400 \mathrm{ft}$ downstream from the confluence of Marsh Creek and Straight Run.

\section{Dry Conditions with 1,000 Gallons per Minute Withdrawals}

Simulations were made for dry conditions with groundwater withdrawals totaling $1,000 \mathrm{gal} / \mathrm{min}$ from the NARL wells. The areas contributing recharge to each well and the configuration of the water table are illustrated in figure $11 \mathrm{~A}$. The area throughout which recharge is captured is more extensive than that for withdrawals of the same magnitude during the wetter "average" conditions of recharge and streamflow shown in figure 6 . The contributing area encompasses more than half the study area, capturing infiltration from multiple sources and directing stream infiltration from both Straight Run and Asaph Run to the wells. Infiltration from Straight Run and Asaph Run accounts for about 49 and 33 percent, respectively, of the groundwater withdrawn by the wells during dry conditions (fig. 11B). Capture from these sources affects the water budget of the study area by decreasing the groundwater discharge to Marsh Creek by about $770 \mathrm{gal} / \mathrm{min}$ (77 percent of 1,000 gal $/ \mathrm{min}$ ) and increasing the total seepage from Straight Run and Asaph Run by about $220 \mathrm{gal} / \mathrm{min}$ (fig. 11C). The ultimate effect on Marsh Creek downstream from the NARL, however, is negligible because the groundwater withdrawals are discharged about $400 \mathrm{ft}$ downstream from the confluence of Straight Run and Marsh Creek.

\section{Dry Conditions with 1,000 Gallons per Minute Withdrawals Plus 1,000 Gallons per Minute Withdrawal from the New Production Well}

Simulations were made for dry conditions with groundwater withdrawals totaling $1,000 \mathrm{gal} / \mathrm{min}$ from the NARL wells and a withdrawal of $1,000 \mathrm{gal} / \mathrm{min}$ from a new well 3,500 ft to the southwest. The areas contributing recharge to each well and the configuration of the water table are illustrated in figure 12A. The contributing area extends throughout nearly all of the study area, capturing infiltration from multiple sources and directing stream infiltration from Straight Run, Asaph Run, and Marsh Creek to the wells. The new well competes only slightly with the NARL wells for infiltration from the headwaters of Asaph Run; thus, the contributing areas for the NARL wells are essentially unchanged (compare figs. 11A and 12A). Infiltration from Straight Run and Asaph Run accounts for about 49 and 33 percent, respectively, of the groundwater withdrawn by the NARL wells during dry conditions (fig. 12B). Induced infiltration from Marsh Creek is not a substantial percentage of the water captured by the wells. The capture by the wells affects the water budget of the study area by decreasing the 

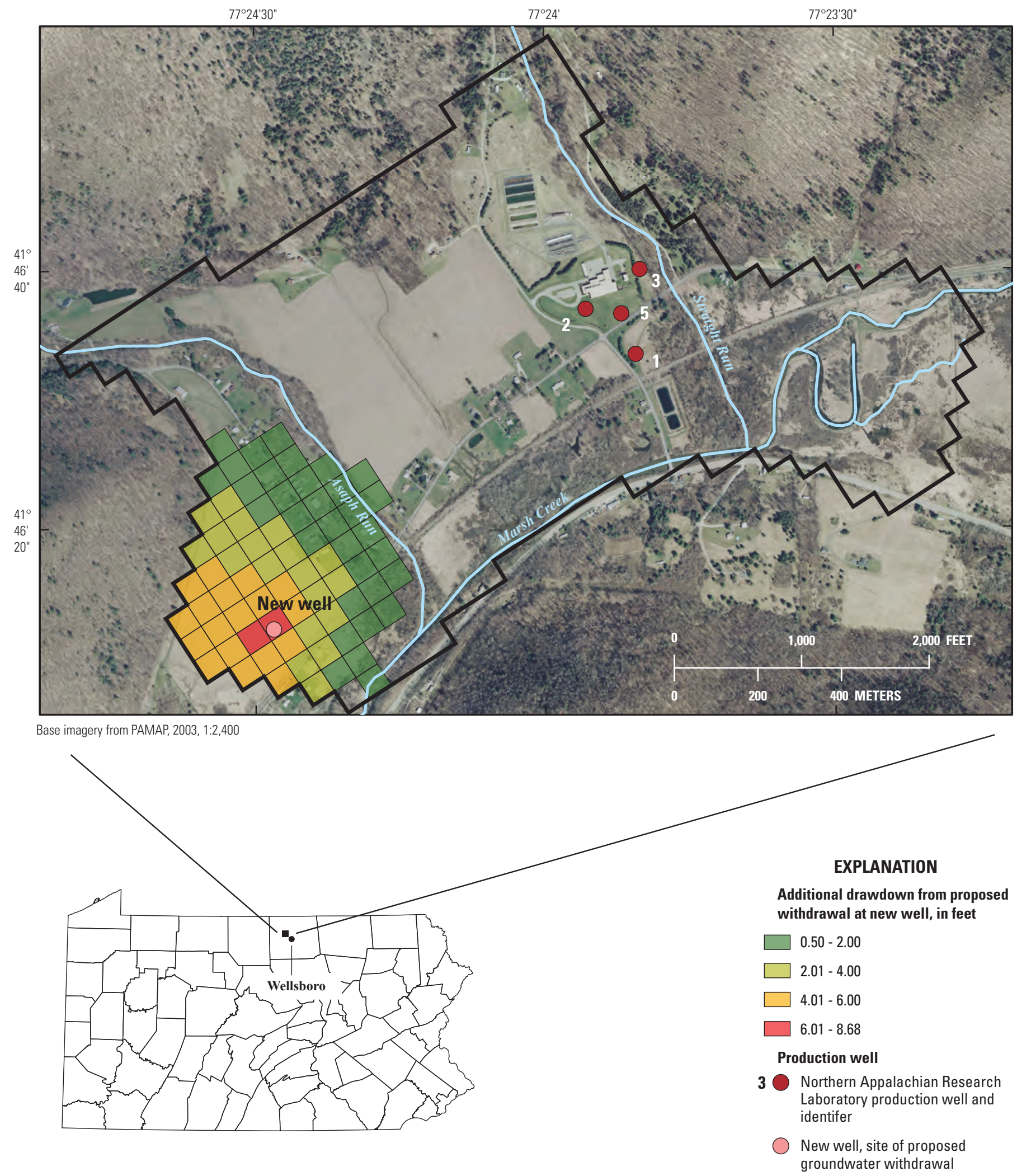

Figure 9. Simulated additional drawdown caused by a proposed groundwater withdrawal of 1,000 gallons per minute southwest of the Northern Appalachian Research Laboratory (NARL) during average hydrologic conditions, Tioga County, Pennsylvania. 


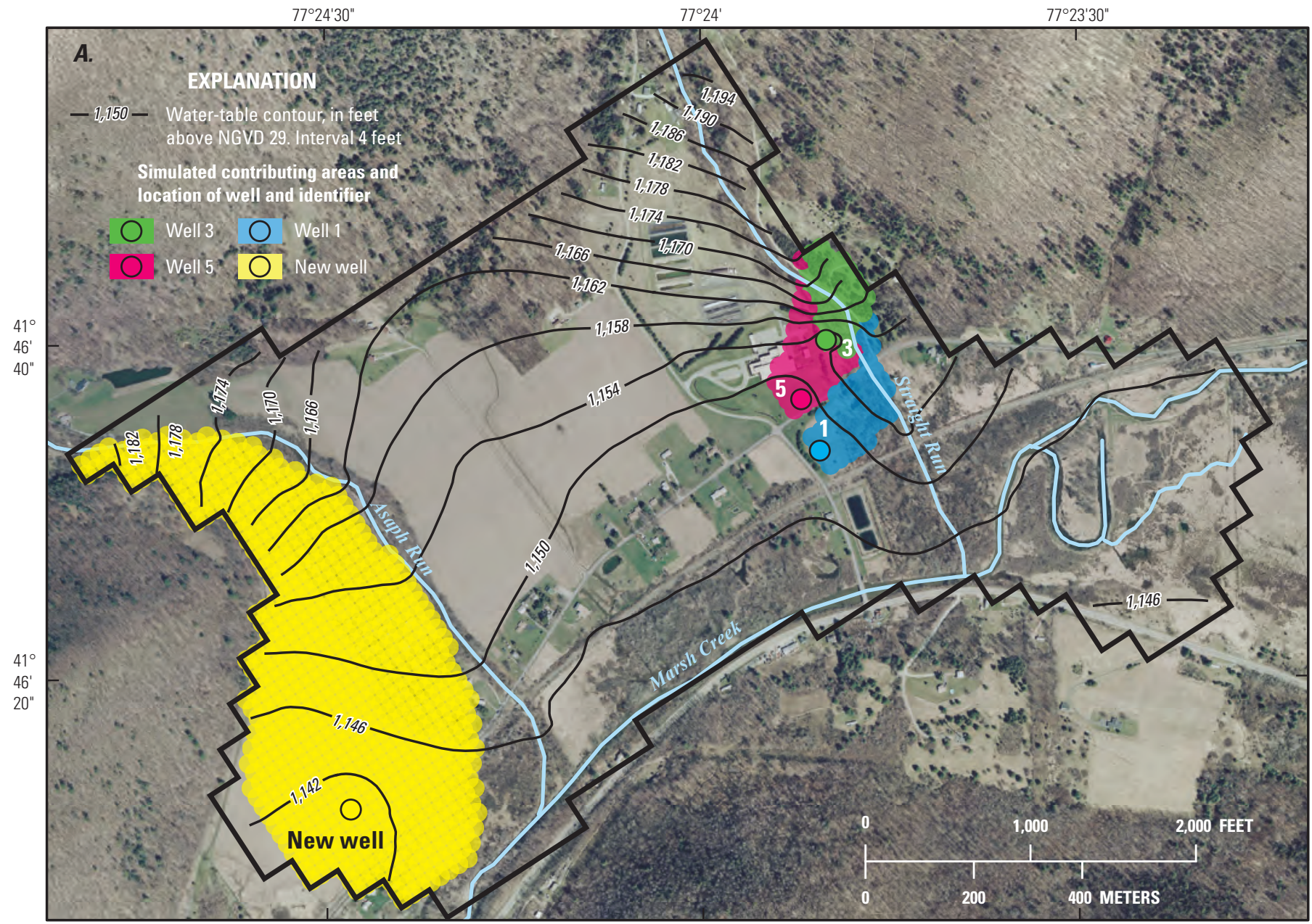

Base imagery from PAMAP, 2003, 1:2,400

\section{B. Sources of water-Northern Appa- lachian Research Laboratory wells}

B. Sources of water-New well

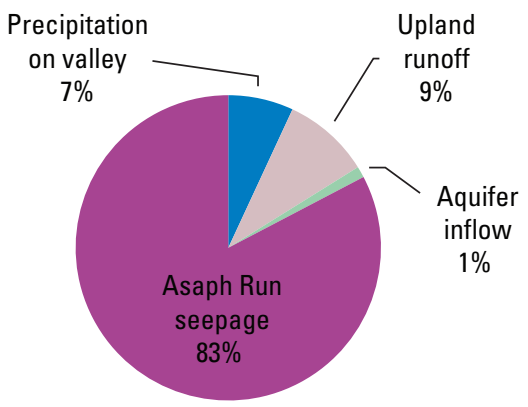

C. Hydraulic response

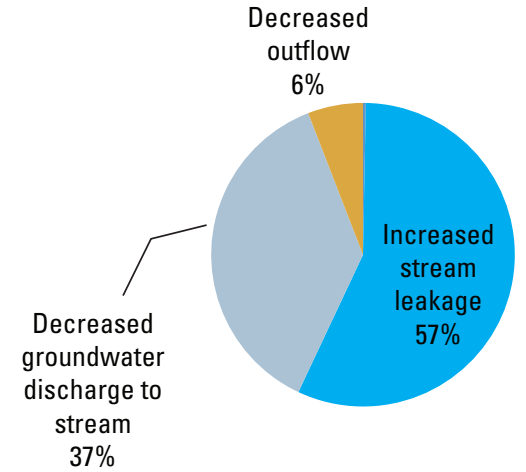

Figure 10. Simulated $A$, areas contributing recharge to wells and water-table contours, $B$, sources of water to wells, and $C$, hydraulic response of the aquifer during average hydrologic conditions with withdrawals of 3,000 gallons per minute from the Northern Appalachian Research Laboratory (NARL) wells and 1,000 gallons per minute from a new production well, Tioga County, Pennsylvania. 


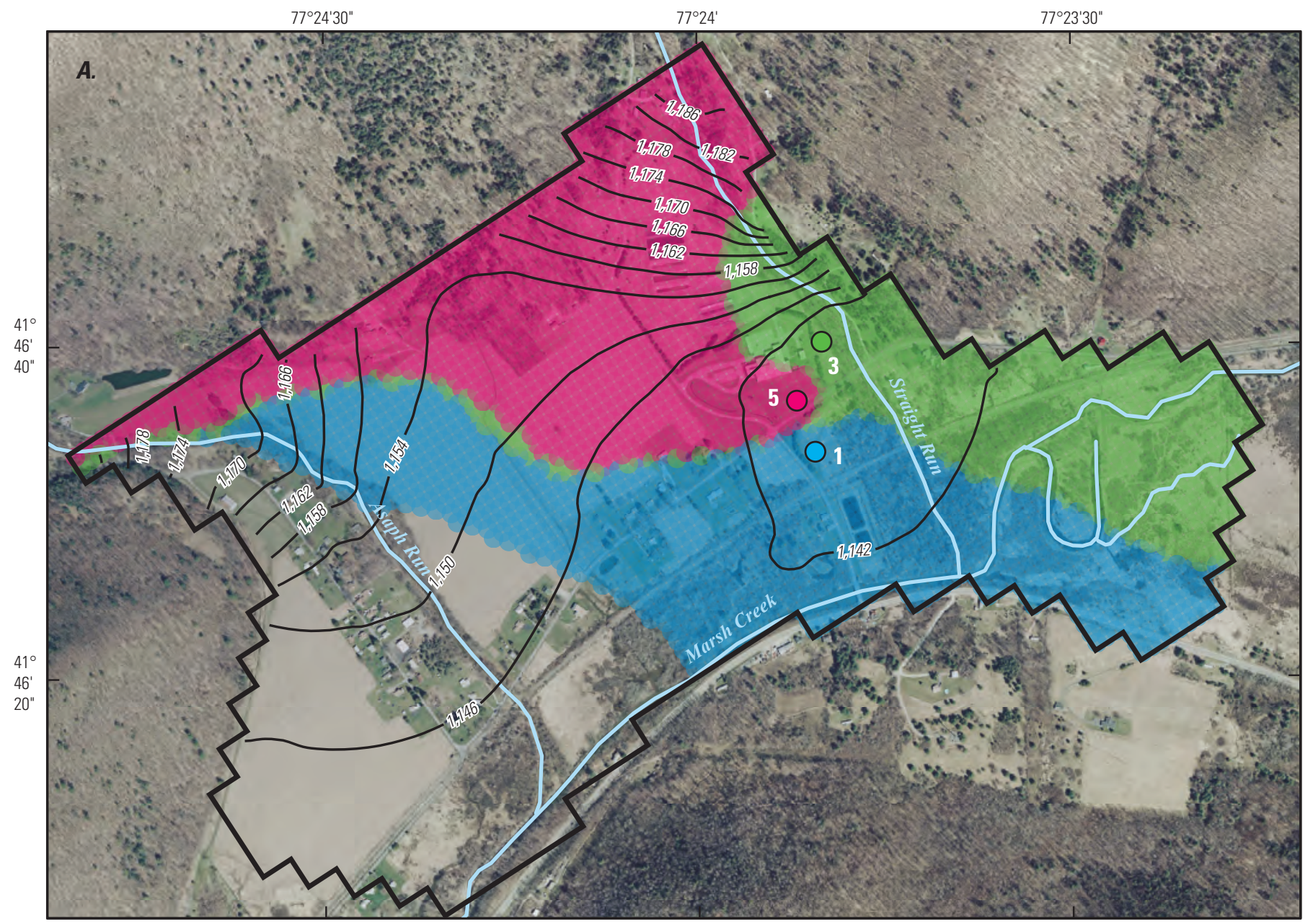

Base imagery from PAMAP, 2003, 1:2,400

\section{B. Sources of water}

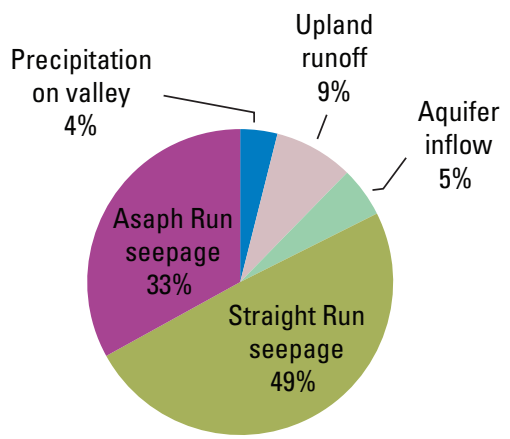

C. Hydraulic response

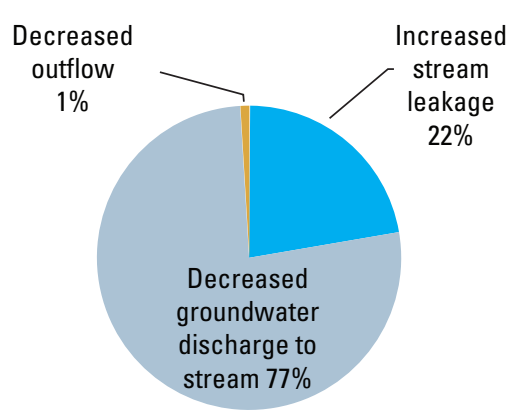

\section{EXPLANATION}

- 1,150 - Water-table contour, in feet above NGVD 29. Interval 4 feet

Simulated contributing areas and location of well and identifier

Well 3

Well 5

Well 1

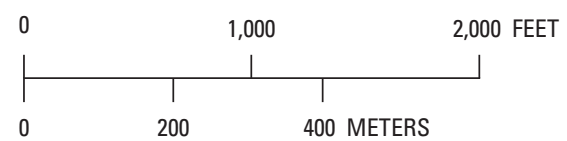

Figure 11. Simulated $A$, areas contributing recharge to wells and water-table contours, $B$, sources of water to wells, and $C$, hydraulic response of the aquifer during dry hydrologic conditions with withdrawals of 1,000 gallons per minute from the Northern Appalachian Research Laboratory (NARL) wells, Tioga County, Pennsylvania. 


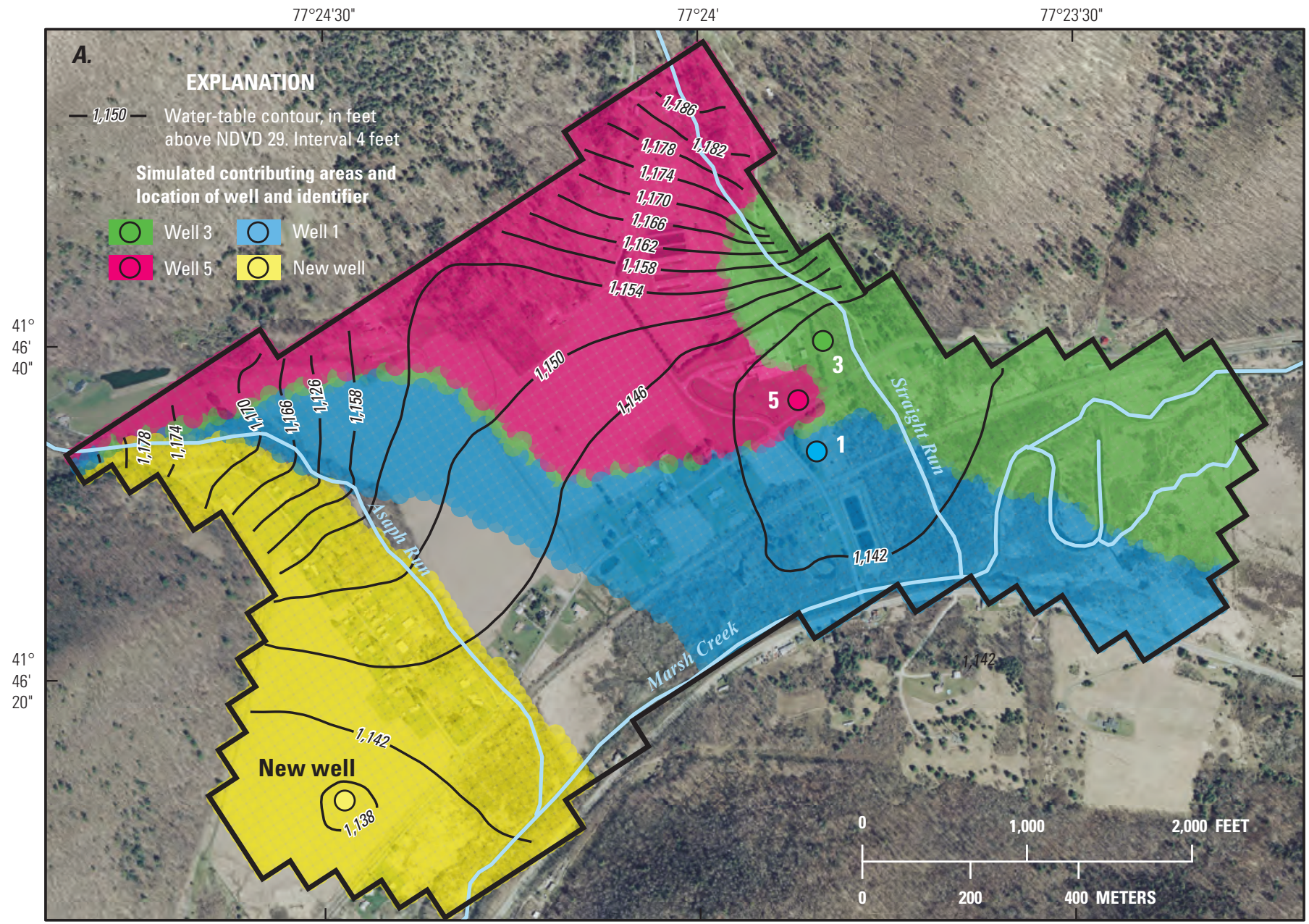

Base imagery from PAMAP, 2003, 1:2,400

\section{B. Sources of water-Northern Appa- lachian Research Laboratory wells}

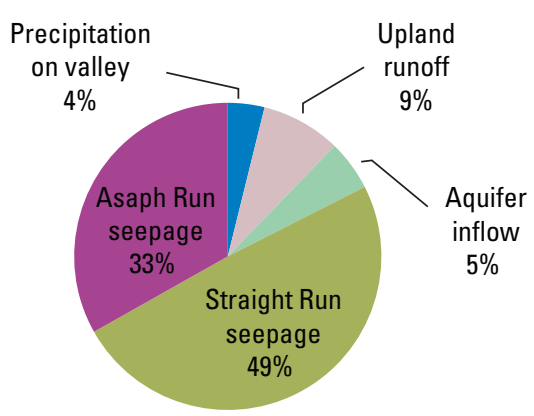

\section{B. Sources of water-New well}

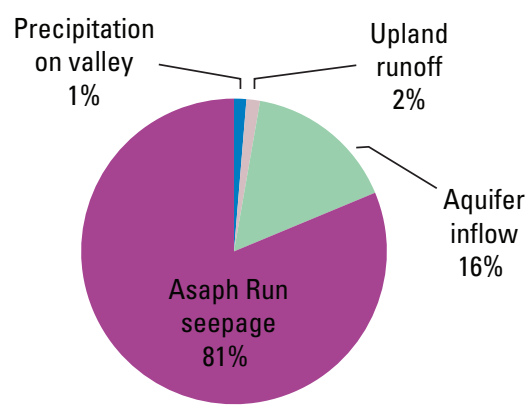

\section{Hydraulic response}

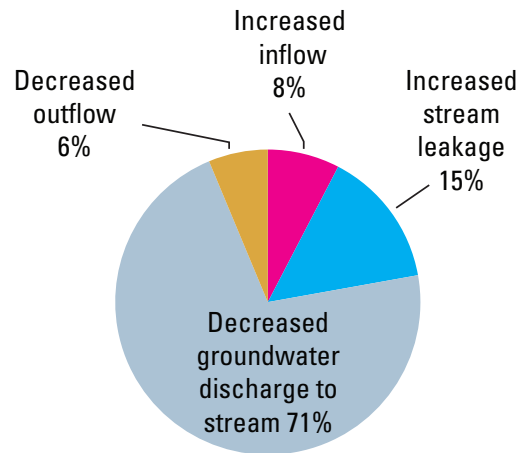

Figure 12. Steady-state simulation of groundwater flow showing $A$, areas contributing recharge to wells and water-level contours, $B$, sources of water to wells, and $C$, hydraulic response of the aquifer during dry hydrologic conditions with withdrawals of 1,000 gallons per minute from the Northern Appalachian Research Laboratory (NARL) wells and 1,000 gallons per minute from a new production well, Tioga County, Pennsylvania. 
groundwater discharge to Marsh Creek by about 1,420 gal/min (71 percent of 2,000 gal $/ \mathrm{min}$ ) and increasing seepage from Straight Run and Asaph Run by about 300 gal/min (fig. 12C). The ultimate effect on streamflow in Marsh Creek downstream from the NARL is caused only by the consumptive withdrawal of $1,000 \mathrm{gal} / \mathrm{min}$ from the new well; withdrawals from the NARL wells are returned as wastewater discharge to Marsh Creek about $400 \mathrm{ft}$ downstream from the confluence of Marsh Creek and Straight Run.

\section{Dry Conditions with 2,000 Gallons per Minute Withdrawals}

Dry conditions were simulated with groundwater withdrawals totaling $2,000 \mathrm{gal} / \mathrm{min}$ from the NARL wells. The areas contributing recharge to each well and the configuration of the water table are illustrated in figure 13A. The area throughout which recharge is captured is more extensive than that for withdrawals of the same magnitude during the wetter "average" conditions of recharge and streamflow shown in figure 7 . The contributing area extends throughout about 80 percent of the study area, capturing infiltration from multiple sources and directing stream infiltration from Straight Run, Asaph Run, and Marsh Creek to the wells. Infiltration from Asaph Run and Straight Run accounts for about 47 and 25 percent, respectively, of the groundwater withdrawn by the wells during dry conditions (fig. 13B). Induced seepage from Marsh Creek supplies about 20 percent of the water captured by wells. The capture of these sources by wells affects the water budget of the study area by decreasing the groundwater discharge to Marsh Creek by about $1,380 \mathrm{gal} / \mathrm{min}(69 \mathrm{percent}$ of $2,000 \mathrm{gal} / \mathrm{min})$ and increasing seepage from Straight Run and Asaph Run by about $600 \mathrm{gal} / \mathrm{min}$ (fig 13C). The ultimate effect on Marsh Creek downstream from the NARL, however, is negligible because the groundwater withdrawals are discharged about $400 \mathrm{ft}$ downstream from the confluence of Straight Run and Marsh Creek.

\section{Dry Conditions with 2,000 Gallons per Minute Withdrawals Plus 1,000 Gallons per Minute Withdrawal from the New Production Well}

Simulations were made for dry conditions with groundwater withdrawals totaling $2,000 \mathrm{gal} / \mathrm{min}$ from the NARL wells and a withdrawal of $1,000 \mathrm{gal} / \mathrm{min}$ from a new well. The areas contributing recharge to each well and the configuration of the water table are illustrated in figure 14A. The area throughout which recharge is captured is more extensive than that for withdrawals of the same magnitude during the wetter "average" conditions of recharge and streamflow shown in figure 8 . The contributing area extends throughout nearly all of the study area, capturing infiltration from multiple sources and directing stream infiltration from
Straight Run, Asaph Run, and Marsh Creek to the wells. The contributing area for the new well competes for water from Asaph Run, thereby causing some changes in the areas contributing recharge to the NARL wells (compare figures 13A and 14A). Infiltration from Straight Run and Asaph Run accounts for about 25 and 45 percent, respectively, of the groundwater withdrawn by the NARL wells during dry conditions (fig. 14B). Induced seepage from Marsh Creek supplies about 21 percent of the water captured by the NARL wells. Seepage from Asaph Run contributes 73 percent of the groundwater withdrawn from the new well. Capture from these sources affects the water budget of the study area by decreasing the groundwater discharge to Marsh Creek by about $1,920 \mathrm{gal} / \mathrm{min}$ ( 64 percent of 3,000 gal $/ \mathrm{min}$ ) and increasing total seepage from Straight Run and Asaph Run by about $780 \mathrm{gal} / \mathrm{min}$ (fig 14C). The ultimate effect on Marsh Creek downstream from the NARL, however, is caused only by the consumptive withdrawal from the new well because groundwater withdrawals from the NARL wells are discharged about $400 \mathrm{ft}$ downstream from the confluence of Straight Run and Marsh Creek.

Model simulations indicate that pumping 1,000 gal/min of water from the new well will cause about $0.5 \mathrm{ft}$ of additional drawdown in the NARL well field during dry hydrologic conditions (fig. 15). However, the magnitude of the drawdown might be greater than the two-dimensional model results indicate because the vertical hydraulic conductivity of the glacial sand and gravel aquifer between the well screen depth and Asaph Run is not simulated by the one-layer model.

\section{Time of Travel}

The contributing areas and sources of water to wells described in the previous section were determined from steady-state model simulations that do not take into account the time required to move water through the contributing zone. In reality, hydrologic conditions do not remain steady forever. Thus, particularly for simulations of dry conditions, a more accurate depiction of the contributing areas and sources of water would be obtained if time of travel were considered. Figure 16 shows the part of the simulated contributing area from which water is captured by the wells during 100 or 200 days of steady withdrawals totaling 2,000 gal $/ \mathrm{min}$ from the NARL wells and $1,000 \mathrm{gal} / \mathrm{min}$ from a new well during dry hydrologic conditions, assuming an effective porosity of 20 percent. Note that the NARL wells do not capture water from Asaph Run even during 200 days of dry conditions, which is a long period for dry conditions to persist.

\section{Assumptions and Limitations of the Model Simulations}

Because the areas contributing recharge and sources of water to wells at the NARL were simulated by the use of a previously documented groundwater-flow model, 


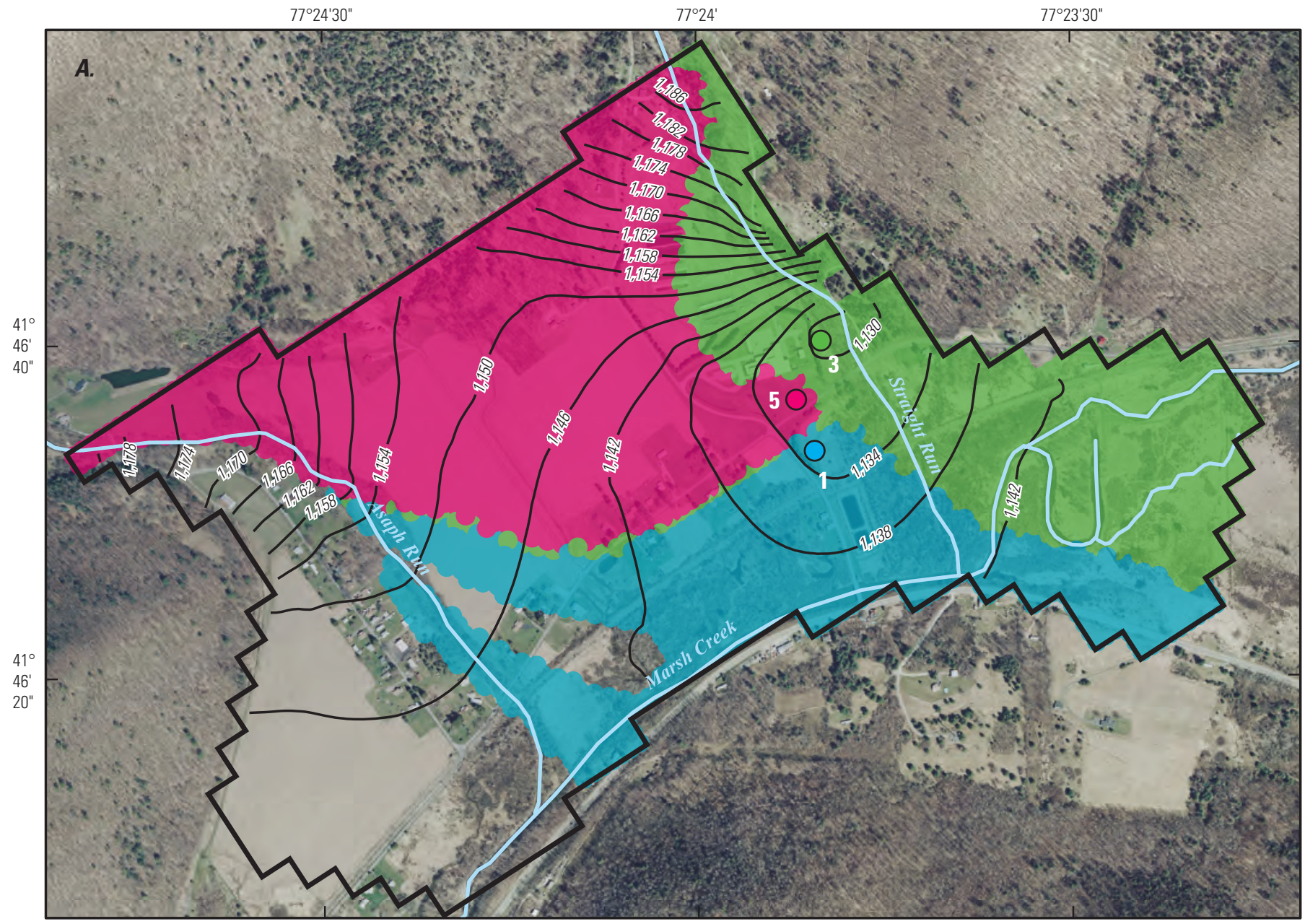

Base imagery from PAMAP, 2003, 1:2,400

\section{B. Sources of water}

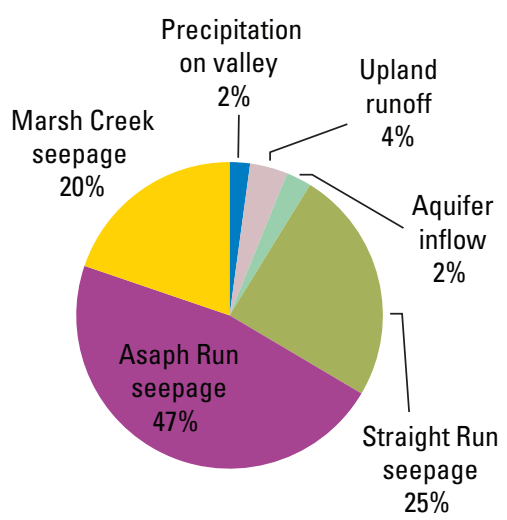

C. Hydraulic response

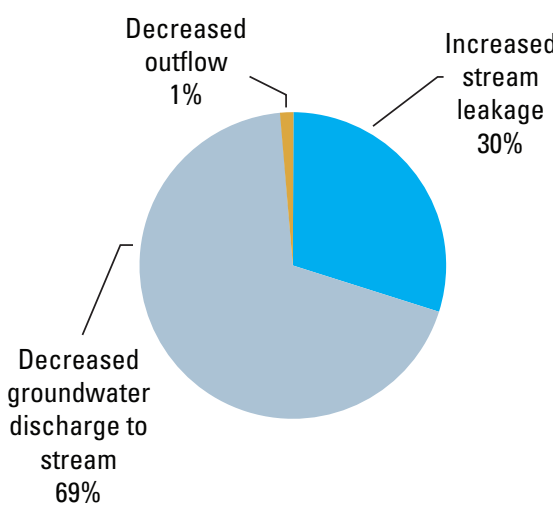

\section{EXPLANATION}

- 1,150 - Water-table contour, in feet above NGVD29. Interval 4 feet

Simulated contributing areas and location of well and identifier

Well 3

Well 5

Well 1

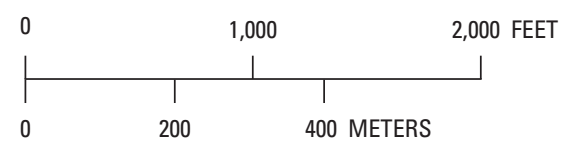

Figure 13. Simulated $A$, areas contributing recharge to wells and water-table contours, $B$, sources of water to wells, and $C$, hydraulic response of the aquifer during dry hydrologic conditions with withdrawals of 2,000 gallons per minute from the Northern Appalachian Research Laboratory (NARL) wells, Tioga County, Pennsylvania. 


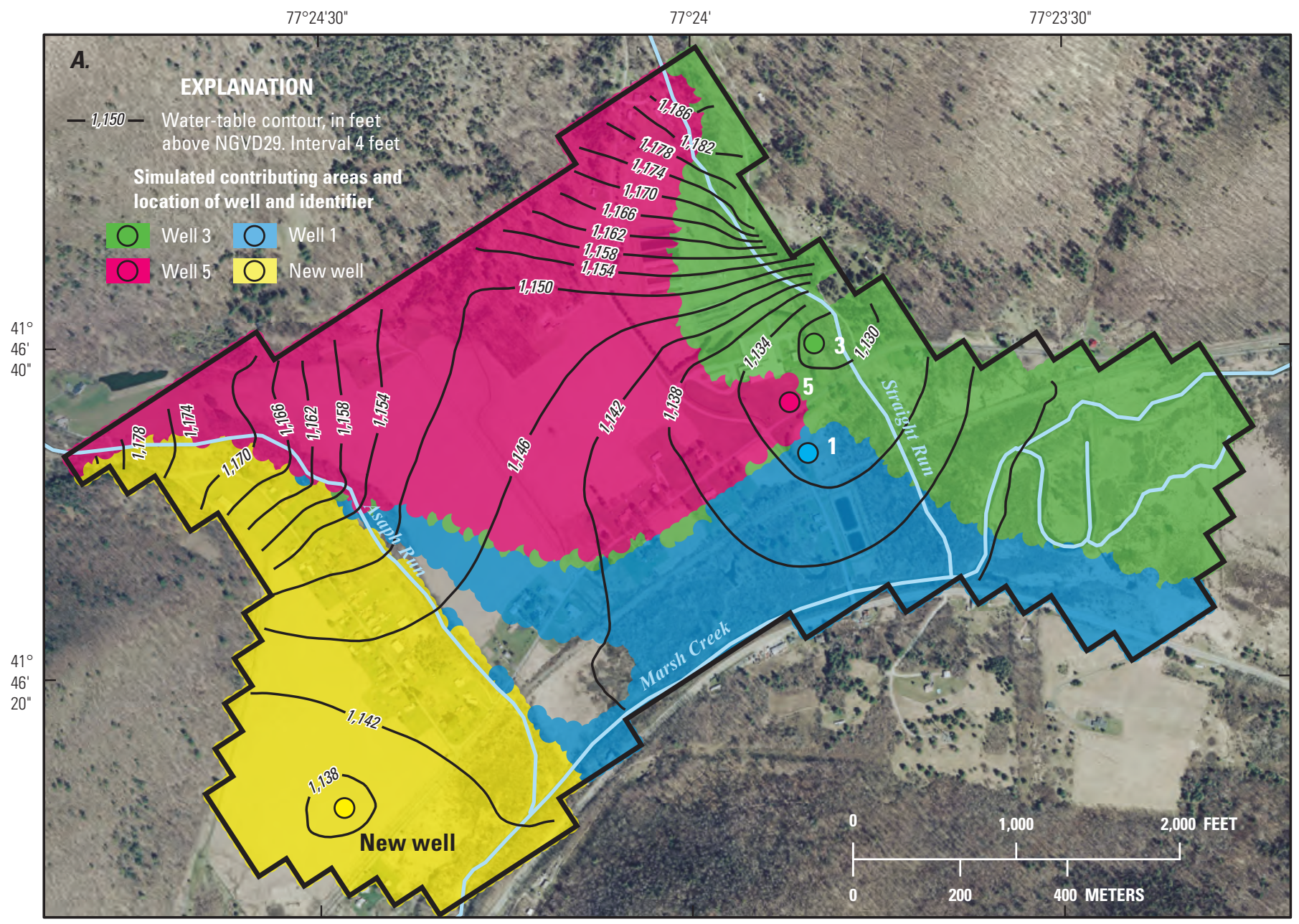

Base imagery from PAMAP, 2003, 1:2,400

\section{B. Sources of water-Northern Appa- lachian Research Laboratory wells}

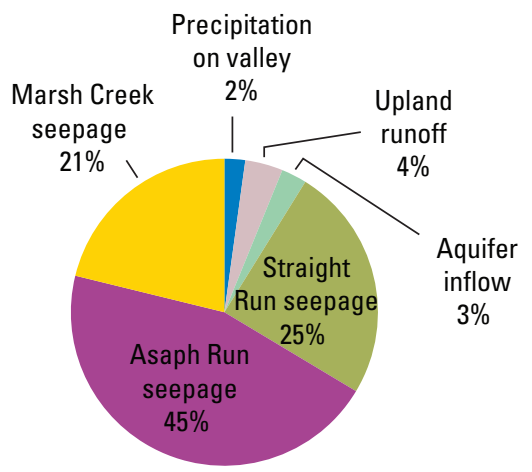

\section{B. Sources of water-New well}

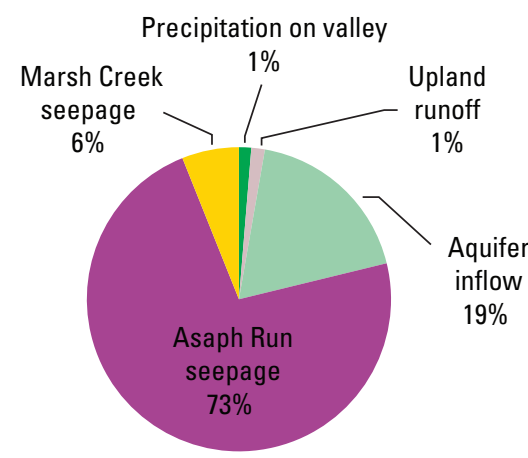

C. Hydraulic response

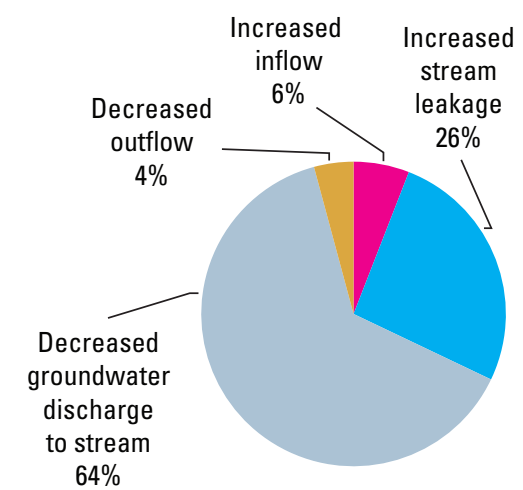

Figure 14. Simulated $A$, areas contributing recharge to wells and water-table contours, $B$, sources of water to wells, and $C$, hydraulic response of the aquifer during dry hydrologic conditions with withdrawals of 2,000 gallons per minute from the Northern Appalachian Research Laboratory (NARL) wells and 1,000 gallons per minute from a new production well, Tioga County, Pennsylvania. 

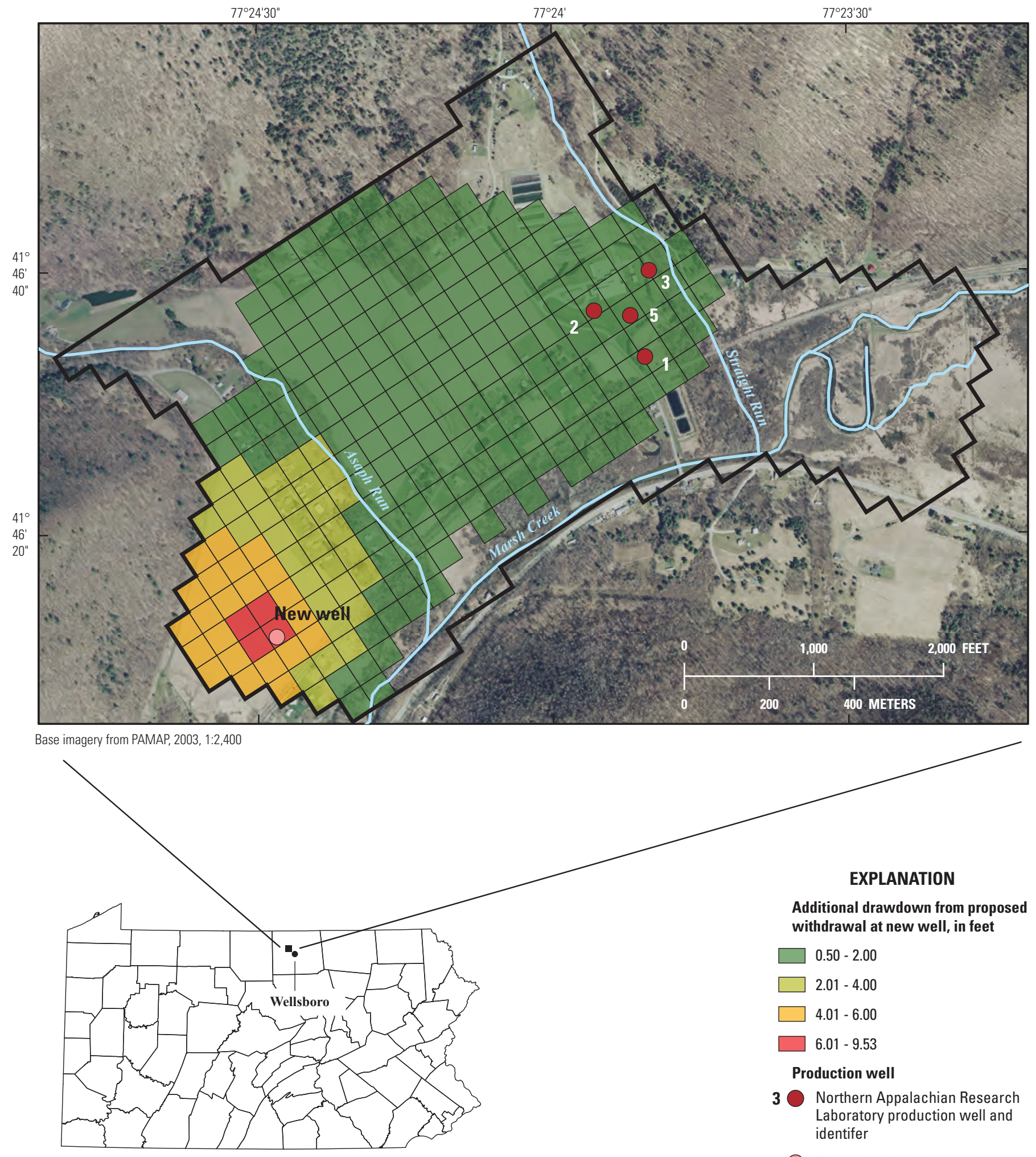

Additional drawdown from proposed withdrawal at new well, in feet

$0.50-2.00$

$2.01-4.00$

$4.01-6.00$

$6.01-9.53$

Production well

3 Northern Appalachian Research Laboratory production well and identifer

New well, site of proposed groundwater withdrawal

Figure 15. Simulated additional drawdown caused by a proposed groundwater withdrawal of 1,000 gallons per minute southwest of the Northern Appalachian Research Laboratory (NARL) during dry hydrologic conditions, Tioga County, Pennsylvania. 

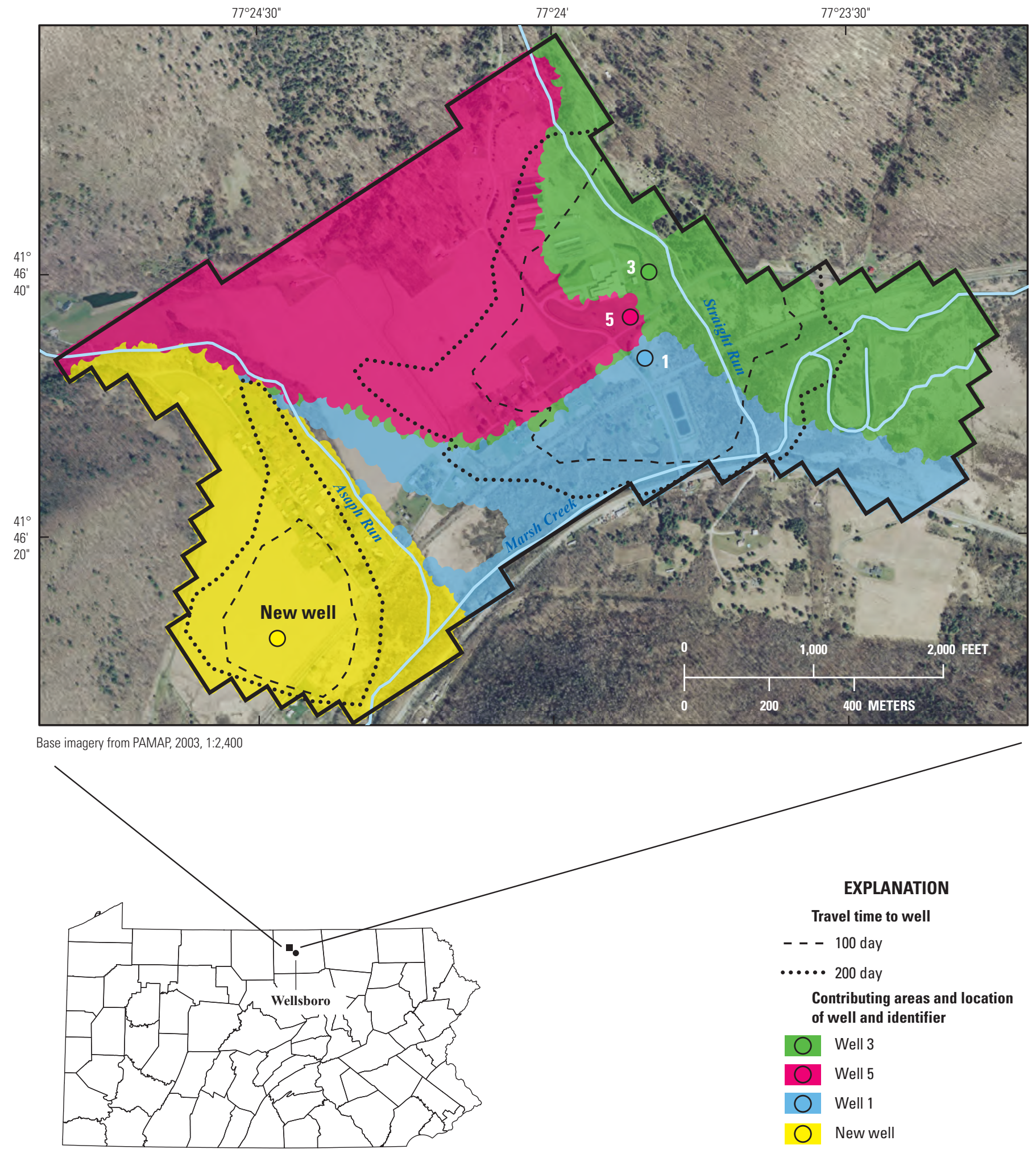

Figure 16. Simulated areas contributing recharge to wells and extent of the areas captured during 100 or 200 days of steady pumping during dry hydrologic conditions in the model area, Tioga County, Pennsylvania. 
the limitations of the model need to be recognized.

The groundwater-flow model of Williams and

Morrissey (1996) used in this study is a two-dimensional (one-layer) representation of steady-state groundwater flow in the glacial sand and gravel aquifer. The model uses a finitedifference grid with cells of $200 \mathrm{ft}$ by $200 \mathrm{ft}$ throughout the model domain and recharge rates specified to represent either average or dry periods. The two-dimensional model is a limitation because vertical differences in boundary conditions and hydraulic properties cannot be incorporated into the model, and vertical hydraulic gradients in the glacial sand and gravel aquifer cannot be simulated. A three-dimensional, multiple-layer model would allow for a more accurate representation of groundwater withdrawals from the wells that are not screened throughout the entire saturated thickness of the aquifer and would allow the vertical hydraulic conductivity to be assigned values typical for stratified sediments. The rather coarse finite-difference grid causes the boundary of the glacial sand and gravel aquifer and well locations to be represented with less accuracy than would be possible if the number of model layers and rows were increased. Smaller cells, combined with more model layers would allow a better simulation of vertical gradients near streams and wells, which could affect the simulated drawdown and the delineation of areas contributing recharge to the wells.

The simulations in this report were based on steady-state conditions of uniform recharge and groundwater withdrawals, although both are known to vary widely. Williams (1991) showed that groundwater recharge to the glacial sand and gravel aquifer varies seasonally, principally on the availability of streamflow from Straight Run and Asaph Run. Thus, although steady-state simulations of dry hydrologic conditions show that it is possible for the NARL wells to capture water from Asaph Run, maps of simulated groundwater time-oftravel indicate that a dry period of unusually long duration would be required. Also, simulations were made for two conditions of steady-state recharge reported in Williams and Morrissey (1996) - termed "average" and "dry" conditions in this report. On the basis of data from the long-term streamgage at Corey Creek near Mainesburg, Pennsylvania, the average recharge probably represents wetter conditions than does the long-term mean recharge, and the dry-condition recharge represents conditions that would happen 2 to 3 months per year. Thus, conditions for a severe drought were not simulated.

In all of the simulations, the sources of water include seepage from streams or runoff that originated from the bedrock uplands. However, because the bedrock aquifers were not explicitly simulated, the areas contributing recharge as illustrated in this study extend only to the boundary of the glacial sand and gravel aquifer along the bedrock valley wall. It should be recognized that capture of stream seepage or upland runoff implies that upland areas outside of the model domain contribute water to the wells.

The new well 3,500 ft to the southwest of the NARL is near the general-head boundary where groundwater exits the glacial sand and gravel aquifer downvalley. Drawdown of 4 to $6 \mathrm{ft}$, caused by groundwater withdrawals from this well, reaches the general-head boundary as does the simulated area contributing recharge to the well. Thus, the properties of this artificial model boundary location probably affect the magnitude of simulated drawdown and might also affect the analysis of the area contributing recharge and sources of water to the well.

\section{Summary and Conclusions}

A study was conducted to document source areas and quality of the groundwater supply at the U.S. Geological Survey (USGS) Northern Appalachian Research Laboratory (NARL) near Wellsboro, Pennsylvania, which is surrounded by the ongoing development of natural gas in the Marcellus Shale. Development of natural gas in the Marcellus Shale poses a potential threat to the quality and quantity of groundwater available to the NARL. Natural gas wells are being permitted and drilled in the Marsh Creek valley and adjacent uplands to the north and south of the valley. A largecapacity water well has been drilled in the glacial sand and gravel aquifer about 3,500 feet southwest of the NARL wells to provide water for a gas company. Land disturbance from access roads, gas wells, and pipeline construction is anticipated, and hydrologic effects from hydraulic fracturing with associated water use and waste handling are possible.

Groundwater samples collected during November 2-4, 2010, from the four wells used by the NARL (tapping the glacial sand and gravel aquifer) and from two nearby domestic supply wells (tapping the Catskill Formation) indicate that the baseline quality of the water is good. The samples were analyzed for major ions, nutrients, trace metals, radiochemicals, dissolved gases, and stable isotopes of oxygen and hydrogen in water and carbon in dissolved carbonate to document groundwater quality. Samples were not analyzed for anthropogenic organic constituents associated with hydraulic fracturing activities, but additional sampling for these constituents would provide a more complete water-quality baseline. Results showed low concentrations of all constituents; only radon, which ranged from 980 to 1,310 picocuries per liter, was somewhat elevated. These findings are consistent with the pristine nature of the aquifer in the Marsh Creek valley, which is the reason the laboratory was sited at this location.

Groundwater modeling simulations showed that the principal source of water to the NARL wells is infiltration from Straight Run with major contributions possible from Asaph Run during dry periods. The sources of water and areas contributing recharge to wells were identified by the use of a previously documented MODFLOW groundwaterflow model for the following conditions: (1) withdrawals of 1,000 to 3,000 gallons per minute from the NARL wells, (2) average or dry hydrologic conditions, and (3) withdrawals of 1,000 gallons per minute from a new well 3,500 feet to 
the southwest of the NARL that was drilled to provide water for Marcellus gas-well operations. Simulations indicated that during average hydrologic conditions, infiltration from Straight Run, a tributary to Marsh Creek, provides nearly all the water to the NARL wells. During dry conditions, the areas contributing recharge expand such that Asaph Run contributes about half of the water to the NARL wells at rates of 1,000 or 2,000 gallons per minute. The addition of a simulated withdrawal of 1,000 gallons per minute from the nearby new well does not substantially affect the sources of water captured by the NARL wells.

The results described in this report have some limitations. The water-quality samples represent only a snapshot of groundwater chemistry for one hydrologic condition; the concentration of some constituents may change temporally. In addition, samples were not analyzed for organic constituents associated with hydraulic fracturing and other human activities. The sources contributing water to the NARL wells and a new well were simulated by use of a simplified one-layer model of the glacial sand and gravel aquifer for steady-state conditions that are never achieved in reality. Although steady-state simulations of dry hydrologic conditions show that it is possible for the NARL wells to capture water from Asaph Run, maps of simulated groundwater time-of-travel indicate that a dry period of unusually long duration would be required. A better analysis could be achieved if the groundwater-flow model were recalibrated with a finite-difference grid having multiple layers, small cells (as small as 10-foot squares near wells), and transient stress periods.

\section{References Cited}

Eltschlager, K.K., Hawkins, J.W., Ehler, W.C., Baldassare, F., 2001. Technical measures for the investigation and mitigation of fugitive methane hazards in areas of coal mining: U.S. Department of Interior Office of Surface Mining Reclamation and Enforcement, Appalachian Regional Coordinating Center, Pittsburgh, Pa., 124 p.

Fishman, M.J., ed., 1993, Methods of analysis by the U.S. Geological Survey National Water Quality LaboratoryDetermination of inorganic and organic constituents in water and fluvial sediments: U.S. Geological Survey Open-File Report 93-125, 217 p.

Fishman, M.J., and Friedman, L.C., eds., 1989, Methods for determination of inorganic substances in water and fluvial sediments: U.S. Geological Survey Techniques of WaterResources Investigations, book 5, chap. A1, 545 p.
Garbarino, J.R., Kanagy, L.K., and Cree, M.E., 2006, Determination of elements in natural-water, biota, sediment, and soil samples using collision/reaction cell inductively coupled plasma-mass spectrometry: U.S. Geological Survey Techniques and Methods, book 5, sec. B, chap. 1, $88 \mathrm{p}$.

Harbaugh, A.W., Banta, E.R., Hill, M.C., and McDonald, M.G., 2000, MODFLOW-2000, the U.S. Geological Survey modular ground-water flow model-User guide to modularization concepts and the ground-water flow process: U.S. Geological Survey Open-File Report 00-92, 121 p.

Hem, J.D., 1985, Study and interpretation of the chemical characteristics of natural water: U.S. Geological Survey Water-Supply Paper 2254, 263 p.

McDonald, M.G., and Harbaugh, A.W., 1988, A modular threedimensional finite-difference ground-water flow model: Techniques of Water-Resources Investigations of the U.S. Geological Survey, book 6, chap. A1, 586 p.

Osborn, S.G., Vengosh, Avner, Warner, N.R., and Jackson, R.B., 2011, Methane contamination of drinking water accompanying gas-well drilling and hydraulic fracturing: Proceedings National Academy of Science, v. 108, no. 20, p. 8172-8176.

PAMAP, 2003, Imagery, 2003, scale 1:2400, accessed February 3, 2011, at http://ceiwin1.cei.psu.edu/ PAMapViewer.html.

Patton, C.J., and Kryskalla, J.R., 2003, Methods of analysis by the U.S. Geological Survey National Water Quality Laboratory_Evaluation of alkaline persulfate digestion as an alternative to Kjeldahl digestion for determination of total and dissolved nitrogen and phosphorus in water: U.S. Geological Survey Water-Resources Investigations Report 03-4174, 33 p.

Pennsylvania Department of Environmental Protection, 2006, Maximum contaminant levels (MCLs) and maximum residual disinfectant levels (MRDLs), accessed March 29, 2011, at http://www.depweb.state.pa.us/portal/server.pt/ community/drinking_water_regulations $\% 2 \mathrm{C}$ _standards resources/10544\#Standards.

Pollock, D.W., 1994, User's guide for MODPATH/ MODPATH-PLOT, Version 3: a particle tracking postprocessing package for MODFLOW, the U.S. Geological Survey finite-difference ground-water flow model: U.S. Geological Survey Open-File Report 94-464, 249 p. 
Prudic, D.E., 1989, Documentation of a computer program to simulate stream-aquifer relations using a modular, finitedifference, ground-water flow model: U.S. Geological Survey Open-File Report 88-729, 113 p.

Risser, D.W., and Madden, T.M., Jr., 1994, Evaluation of methods for delineating areas that contribute water to wells completed in valley-fill aquifers in Pennsylvania: U.S. Geological Survey Open-File Report 92-635, 82 p.

Senior, L.A., 2009, Groundwater-quality assessment, Pike County, Pennsylvania, 2007: U.S. Geological Survey Scientific Investigations Report 2009-5129, 53 p.

Susquehanna River Basin Commission, 2003, Ground-water withdrawal (30-day average) of $1.44 \mathrm{mgd}$ from Well 5, and a total system withdrawal limit (30-day average) of $4.32 \mathrm{mgd}$, for cold-water fish research, Shippen Township, Tioga County, Pennsylvania: Docket No. 20030807, approval date August 14, 2003.

U.S. Environmental Protection Agency, 1999, Proposed radon in drinking water regulation, accessed March 29, 2011, at http://water.epa.gov/lawsregs/rulesregs/sdwa/radon/ regulations.cfm.

U.S. Geological Survey, variously dated, National field manual for the collection of water-quality data: U.S. Geological Survey Techniques of Water-Resources Investigations, book 9, chaps. A1-A9, available online at http://pubs.water.usgs. gov/twri9A.

Wilde, F.D., Radtke, D.B., Gibs, Jacob, and Iwatsubo, R.T., eds., 2004 with updates through 2009, Processing of water samples (version 2.2): U.S. Geological Survey Techniques of Water-Resources Investigations, book 9, chap. A5, April, accessed October 2, 2010, at http://pubs.water.usgs.gov/ twri9A5/.

Williams, J.H., 1991, Tributary-stream infiltration in Marsh Creek Valley, north-central Pennsylvania: U.S. Geological Survey Water-Resources Investigations Report 90-4052, $39 \mathrm{p}$.

Williams, J.H., and Morrissey, D.J., 1996, Recharge of valleyfill aquifers in the glaciated northeast from upland runoff: Subsurface fluid-flow (ground-water and vadose zone) modeling, ASTM STP 1288: Ritchey, J.D., and Rumbaugh, J.O., eds., American Society for Testing and Materials, p. $97-113$.

Winston, R.B., 2000, Graphical user interface for MODFLOW, Version 4: U.S. Geological Survey Open-File Report 00-315, $27 \mathrm{p}$. 
This page has been left blank intentionally. 
Prepared by the West Trenton and Pembroke Publishing Service Centers.

For additional information write to:

Pennsylvania Water Science Center

U.S. Geological Survey

215 Limekiln Road

New Cumberland, PA 17070-2424 
